D.

DESY $95-17 T$

September 1995

The H1 Backward Calorimeter BEMC and its Inclusive Electron Trigger

H1 BEMC GROLP

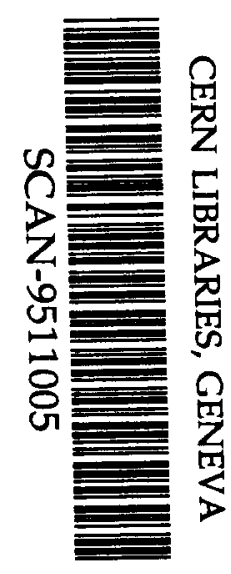

$\sin 9545$ 


\title{
The H1 Backward Calorimeter BEMC and its Inclusive Electron Trigger
}

J. Bán ${ }^{6}$. W. Bauhoff ${ }^{4}$ D. Bruncko ${ }^{6}$. ( Brune $^{5}$. F. Claaßen ${ }^{4}$. H.H. Duhm ${ }^{4}$, E. Eisen ${ }^{4}$.

M. Eschweiler ${ }^{4}$.J. Ferencei ${ }^{5}$. M. Fleischer ${ }^{2}$. W. Gärtner ${ }^{4}$. M. Gennis ${ }^{4}$. A. Cilazor.

R. Griebel ${ }^{4}$. C. Gnelck ${ }^{4}$. M. Harning ${ }^{4}$. T. Hartmann ${ }^{4}$. E. Hoelzke ${ }^{5}$. M. Javorek ${ }^{6}$.

H.-P. Kasselmann ${ }^{4}$. M. W. Krasny. . F. Kriván ${ }^{6}$. H. Krause ${ }^{4}$. J. Koch ${ }^{4}$. I. Kühn ${ }^{4}$

T. Kurča ${ }^{6}$. R. Langkan ${ }^{4}$. M. Lipka ${ }^{4}$. R. Maracek ${ }^{6}$ M. Matysek ${ }^{4}$. K. Meier ${ }^{5}$. P. Murn ${ }^{6}$.

T. Novák'. J. Olszowstial. F. Peppel. ('. Pichler". K. Rathje . P. Reimer".

S. Reinshagen ${ }^{3}$. W. Scobel ${ }^{4}$. N. Schirm ${ }^{4}$. ('. Schrader ${ }^{4}$ S. Schrieber ${ }^{4}$. M. Semant". P. Skvaril' . J. Spalekt. R. Wunderlich ${ }^{4}$. D. Zarbock ${ }^{4}$

Institute for Nuclear Physics. (racow. Poland

2 Institut für Physik. Iniversität Dortmund. Dortmund. Germany

${ }^{3}$ Deutsches Elekt ronen Synchrotron. Hamburg. Germany

${ }^{4}$ I. Institut für Experimentalphysik, Vniversität Hamburg. Hamburg. Ciermany

"Institut für Hochenergiephysik. Iniversität Heidelberg. Heidelberg. Germany

"Institute of Experimental Physics. Slovak Academy of Sciences. Košice, Slovak Republic

' Institute of Physics. ('zech Academy of Sciences. Prahia. Czech Republic

${ }^{8}$ DFSY. Institut für Hochenergiephysik. Zeuthen. Germany

\begin{abstract}
:
A sandwich type lead-scintillat or electromagnetic calorimeter with wavelength shifter optical readout has been successfully operated at the DESY $e p$ collider HERA in the H1 detector for three vears. The mechanical design of the calorimeter together with the associated electronics and the inclusive electron trigger as well as its performance and stability in test beams and at the $r p$ collider HERA are described in detail.
\end{abstract}




\section{Introduction}

The Hl experiment is a general purpose detector [1] which employs magnetic spectroscops. calorimetry and muon detection for electron-proton physics at HERA. It has been operational since 1992. The large liquid argon calorimeter system [2] is supplemented by warm calorimeters covering the smaller scattering angles with respect to the incoming beams.

HERA provides access to a new regine of four monent um transfer with $Q^{2}$ extending up to values of more than 50000 (iel" 10 probe the structure of the proton. Such deep inelastic scattering (DIS) processes are characterized by electrons emitted at large angles into the acceptance of the $\mathrm{H} 1$ liquid argon calorimeter system. Smaller electron scattering angles corresponding to four momentum transfers in the range 5 Gel-2 $<Q^{2}<100$ Ciel- are covered by a conventional lead-scintillator sandwich calorimeter. The primary task of this backward electromagnetic calorimeter (BEM(') is to trigger on electrons and to measure their energy and position. In addition the BE.VC has to contribute to the reconstruction of hadronic final states emerging from DIS at low values of the Bjorken-x variable and from photoproduction processes.

In this paper we give a detailed description of the mechanical layout. the electronics sustem. the inclusive electron trigger. the calibration concept and the performance of the BEMC' in electron-proton collisions at HERA.

\section{Detector description}

\subsection{Environment}

The BEMC is mounted inside the $H 1$ solenoid coil at a distance of $z=-144 \mathrm{~cm}$ (calorimeter front face) from the interaction point (IP). The negative $z$-axis of the $\mathrm{H} 1$ coordinate system is defined to point along the electron direction. The solenoidal field at this location is approximately 1.0 T on the z-axis. Radial and longitudinal inhomogeneities of the field close to the end of the coil are still less than 8,7 at this position. 'The BEMC' covers polar angles $\theta$ in the range $151^{\circ}<\theta<176^{\circ}$ and gives full azimuthal coverage for particles emerging from the nominal interaction point. This corresponds to approximately 1.3 units of pseudorapidity $\eta$ in the range $-2.9<\eta<-1.6$.

An overlap region between the most backward part of the liquid argon calorimeter (BBE) and the BE.MC in the angular range $151^{\circ}<\theta<155^{\circ}$ prevents particles from escaping detection between the two calorimeter sistem. Tracking of charged particles is provided by the backward multiwire proportional chamber (BP(') mounted directly on the calorimeter surface with an angular acceptance $1.55^{\circ}<\theta<177^{\circ}$. and by the central tracking system of the $\mathrm{H}$ l detector measuring track elements and momenta for scattering angles $\theta<170^{\circ}$. The four planes of the BP(' measure the impact position of charged particles hitting the BEMC with a precision of $1.5 \mathrm{~mm}$. Approximately 1 radiation length $X_{0}$ of passive material from support structures and cables is between the central 1 racking system and the combined BEMC/BPC systent. Downstream in the electron direction the BEMC is followed by a time-of-flight system (ToF). which covers roughly the same acceptance. This detector is used to veto proton induced beam wall and beam gas background events by virtue of their early time of arrival compared with genuine cp collisions. Fig. 1 gives an overview of the $\mathrm{H} l$ backward area.

The event rates expected in the BLMC region from genuine $\epsilon$ interactions are moderate. Even at the HERA design luminosity of $1.5 \times 10^{31} \mathrm{~cm}^{-2} \mathrm{~s}^{-1}$ DIS processes with the electron scat tered into the BEMC' do not exceed $5 \mathrm{~Hz}$. In practice the event rate in the BLX(' is dominated by accelerator induced background events originating mainly from the proton beam and 


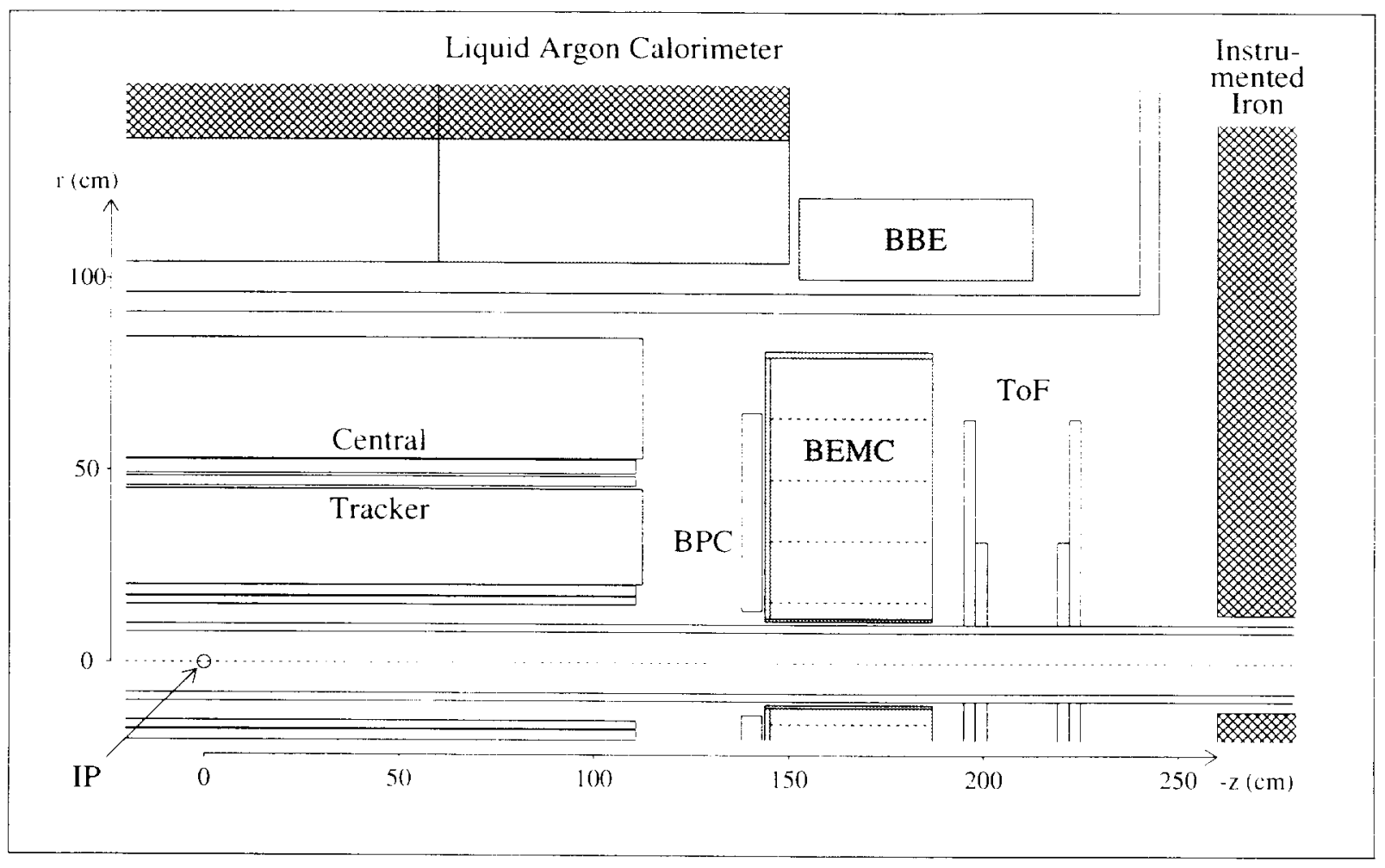

Fig. 1: Longitudinal cross section of the backward region of $\mathrm{H} 1$ detector.

synchrotron radiation from the electron beam. The rate of hadronic proton interactions with gas molecules in the beam pipe and with machine elements like vacuum pumps and collimators can be as high as $10 \mathrm{kHz}$ during stable luminosity runs. These conditions represent a major challenge for analog signal processing and triggering on inclusive electrons. This hadronic background gives rise to radiation doses. which during the years 1992 - 1994 amounted to approximately $200 \mathrm{~Sv}$ integrated dose.

\section{$2.2 \quad$ Mechanical design}

The BEMC consists of $x x$ calorimeter modules. called stacks. which are mounted in a cylindrical aluminium barrel. The transverse view is shown in Fig. 2a. The aluminium barrel covers a radial range $10.8 \leq r \leq \lambda 1.1 \mathrm{~cm}$ surrounding the beampipe. The stacks are arranged in 10 rows and 10 columns inside the barrel and are supported at their front and back. Each stack is fixed by a pin mounted on the stack front plate. which fits into a hole of the same diancter in the barrel front plate. The backs of the stacks are fixed by supporting girders. The total weight of the BEMC is $3600 \mathrm{~kg}$. which is supported by the cryostat of the liquid argon calorimeter.

56 of the 88 stacks have a square cross section of $15.9 \mathrm{~cm} \times 15.9 \mathrm{~cm}$. The cross sections of the remaining 32 stacks are chosen to be trapezoid or triangular so that the outer and inner regions of the barrel are instrumented in the most effective way.

Viewed from the $\mathrm{H} 1$ interaction point cath stack (Fig. 2) consists of an $8 \mathrm{~mm}$ thick aluminium front plate with a pin on top. a first $4 \mathrm{~mm}$ scintillator plate followed by 49 sampling lavers composed of $2.5 \mathrm{~mm}$ lead $\left(\mathrm{PbSb}_{\mathrm{f}}\right) .0 .2 \mathrm{~mm}$ paper. $4 \mathrm{~mm}$ scintillator and $0.2 \mathrm{~mm}$ paper adding up to 21.7 radiation lengths $X_{0}$. The paper sheets are used to improve the optical response. They have stack type dependent grey patterns 10 increase the homogeneity of the signals. The end plate $(8 \mathrm{~mm}$ aluminium, followed by the electronics housing fixes the sampling structure inside stainless steel boxes which have thin walls of $0.2 \mathrm{~mm}$. 


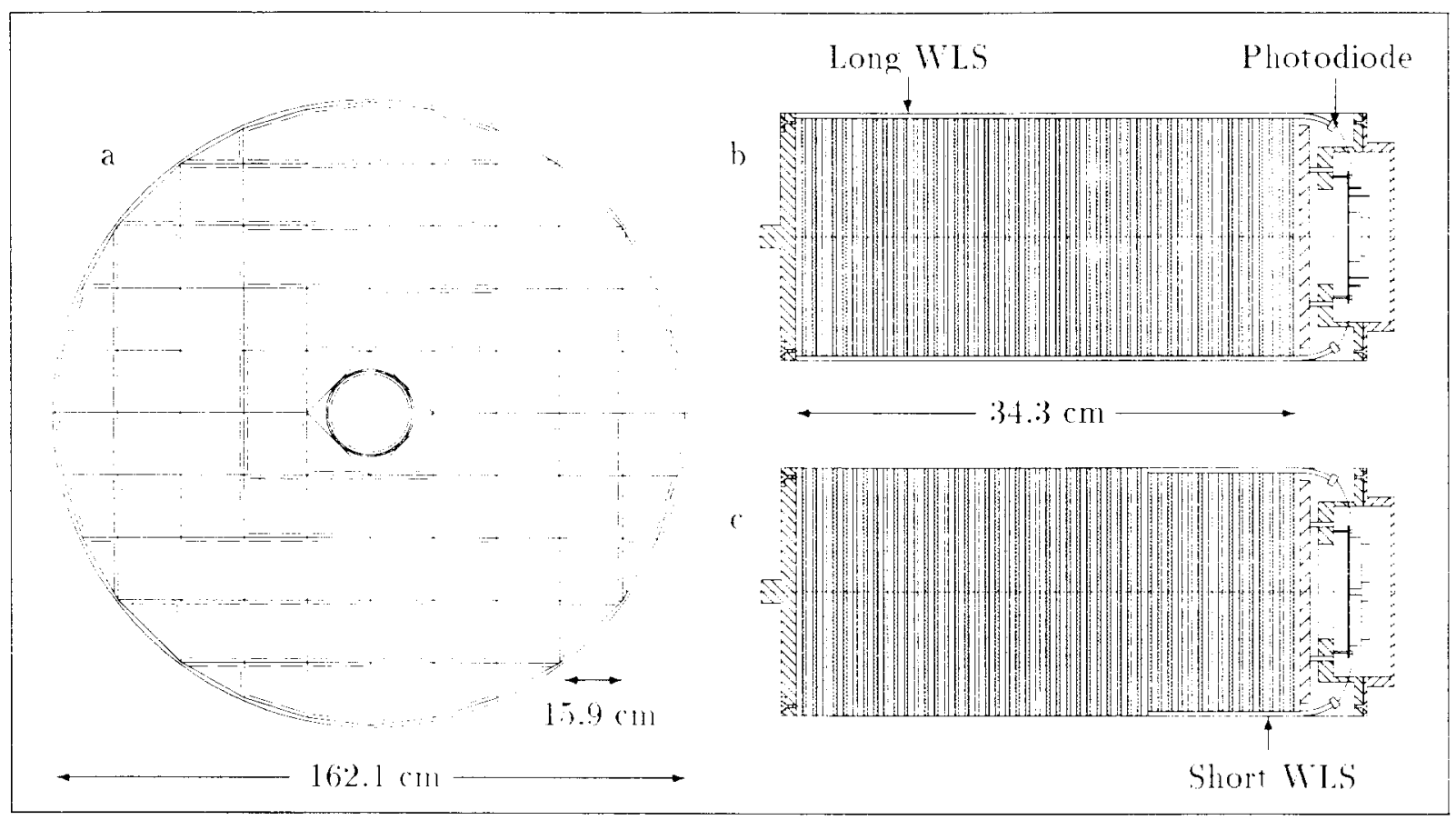

Fig. 2: Transverse view of the BEMC barrel and longitudinal cross sections of BEMC stacks. The positions of all long WLS in the BEMC are marked in (a). The scintillating light is read out transversely via long WLS covering the full length of BE.IC stacks (b). In square and trapezoid stacks the last 15 sampling layers are read out via short WLS (c).

The scintillator type used in the sampling structure is $5 \mathrm{CSN}-38$ [3]. The primarily ultraviolet scintillation light produced by passing particles is shifted by dye to the blue region $(400-450 \mathrm{~nm})$ and read out via wavelength shifters (WLS) extending over the full depth of each stack. The WLS material is Y-T [3]. The light emitted by the WISS is in the range between 450 and $5.50 \mathrm{~nm}$ which is received by photodiodes glued to the end face of each WLS bar.

The four WLS bars with a cross section of $3 \mathrm{~mm} \times$ it $\mathrm{mm}$ extending over the full depth of a stack are referred to as "long. WIS. They are mounted on two opposite sides of a BEMC stack. The arrangement of all long WLS is shown in Fig. 2a. The orientation of the stachs is optimized for particles coming from the interaction point such that the path length through the regions of the WLS is minimal. For all square stacks and large trapezoid stacks the scintillating light of the last 15 sampling layers is read out by separate (short) WLS. The short WLS are twice as broad as the long WLS. Each long WLS is coupled to one photodiode whereas the short WLS are coupled to two photodiodes. In order to prevent direct optical contact between scintillator plates and WLS bars nylon threads of $0.3 \mathrm{~mm}$ diameter are used to separate the WLS from the scintillator. At the rear region near the photodiodes the WLS - which are $25 \mathrm{~mm}$ longer than the sampling structure covered are slightly bent to the center of the stack. This is necessary for suppression of direct semiconductor counting effects in the photodiodes that are produced by particles passing directly through the photodiodes. Each WLS is covered by white reflecting paper to achieve a better optical response. The sheets of reflecting paper covering the long WLS have grey patterns to increane the longitudinal homogeneity of the signals.

Each WLS is assigned to one electronic channel. There are 344 long WLS channels and $12 \mathrm{x}$ short WLS channels resulting in ti2 readout channels in total.

For functionality test and monitoring purposes a laser monitor system has been developed

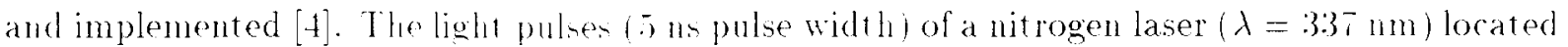


outside the $\mathrm{H} 1$ detector are transported via two quartz glass fibers (core diameter $1 \mathrm{~mm}$ ) to the BEMC area, where they are distributed into two bundles of secondary quartz glass fibers. These secondary fibers have a core diameter of $0.2 \mathrm{~mm}$. Two secondary fibers. one from each bundle. are connected to each stack at two points to the last scintillator plate (only one fiber for small triangular stacks). Four additional fibers. two from each bundle. are connected to separate diodes to be used for reference purposes.

\section{$2.3 \quad$ Electronics}

The task of the electronics chain is to convert the light transmitted by the WIS into electric charge to amplify the signal. 10 extract a fast trigger. to perform analog signal processing and to digitize the signal for readout.

Photodiodes have been chosen as photon detectors for the BEMC because of their excellent intrinsic stability and their undistorted performance in large magnetic fields. The necessary charge amplification by electronic circuits. however. gives rise to rather slow signals compared to photomultipliers and to electronic noise. The BEMC' is read out by silicon PIN diodes of type Hamamatsu S25i5[5]. Their sensitive surface is $30 \times 3.4 \mathrm{~mm}^{2}$. Their broad spectral sensitivity in the range $320 \mathrm{~nm}<\lambda<1060 \mathrm{~nm}$ is well matched to the emission spectrum of the WLS. The diodes are operated at a bias voltage of $40 \mathrm{~V}$ corresponding to a depletion zone of $130 \mu \mathrm{m}$ thichness and a capacitance of $80 \mathrm{pF}$. Typical dark currents are $5 \mathrm{nA}$. A 1 GeV electron in the BEMC corresponds to a photodiode signal of 4260 photoelectrons giving rise to a signal of $8.5 \mu \mathrm{V}$. Each diode is connected to a charge sensitive preamplifier. The amplifier increases the signal by a factor 100 with an equivalent noise charge of 48.3 electrons. The actual signal-to-noise ratio is improved by the feature of reading out each calorimeter stack with four independent WLS-photodiode systems. The rise time of the preamplifier output signal is $100 \mathrm{~ns}$ with a fall time reaching $1.50 \mu \mathrm{s}$. The preamplifiers are mounted on a motherboard directly on the calorimeter stacks. Test charges can be injected into the preamplifiers in order to calibrate the response of the entire electronics chain including cables. The preamplifiers are followed by differential line drivers transmitting the analog signals via $30 \mathrm{~m}$ long twisted pair cables to an electronics hut outside the $\mathrm{H} 1$ detector. The entire electronics installed on the calorimeter stacks dissipates a power of $350 \mathrm{~W}$ which is carried away by a water cooled copper shield mounter on the back of the calorimeter.

The differential pulses arriving through the twisted pair cables in the electronic hut are split to provide inputs for the calorimetrie triggers and for the readout. Two trigger signals are produced. First the analog signals of the four long WLS bars within one stack are smmmed in order to provide inpul for the high granularity inclusive electron trigger (see next section). A second set of stack sums in formed to feed the overall calorimeter trigger sums with lower granularity. The timing and the gain of the BEMC inclusive trigger stack sums are individually adjusted in order to assign events uniguely to one HER I bunch crossing and to compensate for stack-to-stack variations in energy calibration.

The analog processing of individual readout channels proceeds through a chain of shapers. sample-and-hold and multiplexing circuits. The basic problem at machines with large bunch crossing frequencies like HERA is that a trigger decision is only available after many bunch crossings. The decision time of the first level trigger in $\mathrm{HI}$ is 25 bunch crossings. corresponding 102400 ns. Due to the large cross section of calorimeter stacks and the high proton-beam related background rates the analog signal processing has to be carried out much faster. The probability of a distortion of the energy measurement through pile-up in this time interval would exceed $50 \%$ for a background rate of $100 \mathrm{kHz}$ corresponding to most pessimistic background expectations. To overcome this problem an analog electronics chain consisting of a fast. unipolar shaper and a subsequent analog delay line is used. The shaper has a time constant of $450 \mathrm{~ns}$ (FWH.M) in order 
to reduce the pile-up window 10 about 5 HERA bunch crossings. In order to match the trigger decision time the shaped signal is delayed by an analog delay line with a length of $2.4 \mu s[6]$. To compensate for tolerances in the length of the delay line and the overall signal propagation time in the analog electronics the delay line has been equipped with 10 taps to adjust individual delays in steps of $20 \mathrm{~ns}$ in the range from $2.2 \mu \mathrm{s}$ to $2.4 \mu \mathrm{s}$. The fast shaping requires a precise relative adjustment of all tiz delay lines in order to avoid channel-to-channel gain variations of the held signal. Equalization of the delay lines to $60 \mathrm{~ns}$ is required to keep the gain variationwithin $1 \%$. The result of the equalization can be seen in Fig.3. The channel-to-channel delay variation has a spread of 9.8 us (rms).

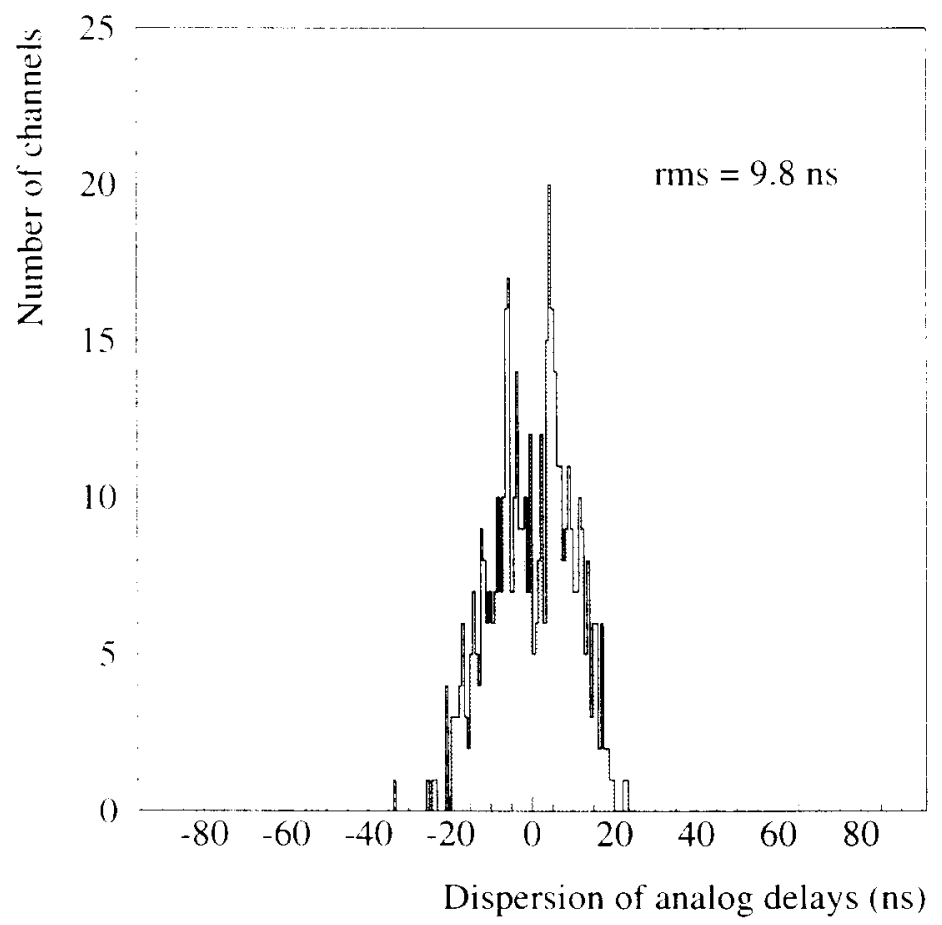

Fig. 3: Distribution of individual delạy line timings of all BEMC readout channels.

16 analog channels are combined on 1 card providing a single multiplexed output of the held voltages. $s$ analog cards are combined into 1 crate with a further multiplexing by a factor $x$.

The analog voltages are digitized in 12 bit $A D$ ('s. Pedestal subtraction. noise suppression and a nonlinear online calibration based on a third order polynomial are carried out on a dedicated processor [2].

In order to maintain the required precision. it is necessary to correct for different gains and nonlinearities of individual electronic channels. This is determined by a precise meanurement of the ADC-response to a known elect ronic pulser signal. For each channel $j$ a set of parameters $P_{i, j}$ is determined on the basis of calibration measurement (ADC response vs. pulser signal) by fitting a third order polynomial. This set of channel dependent parameters including the value of the pedestals $\left(P \in d_{j}\right)$ in uned online during the data acquisition to correct for pedestals and nonlinearities using the formula $E_{j}=\sum_{i=1,3} P_{i, j} \times\left(A D C_{j}^{\prime}-P \in d_{j}\right)^{i}$.

Fig. 4 shows the stability of the complete electronic chain over the whole run-period in 1992 (2 month duration) for a pulser level which corresponds. for channels comected to long WISS in square stacks - roughly to a deposited energy of 30 Gel. The stability is better than $0.1 \%$ and even this has been corrected on the basis of the electronic calibration by using the above mentioned formula. 


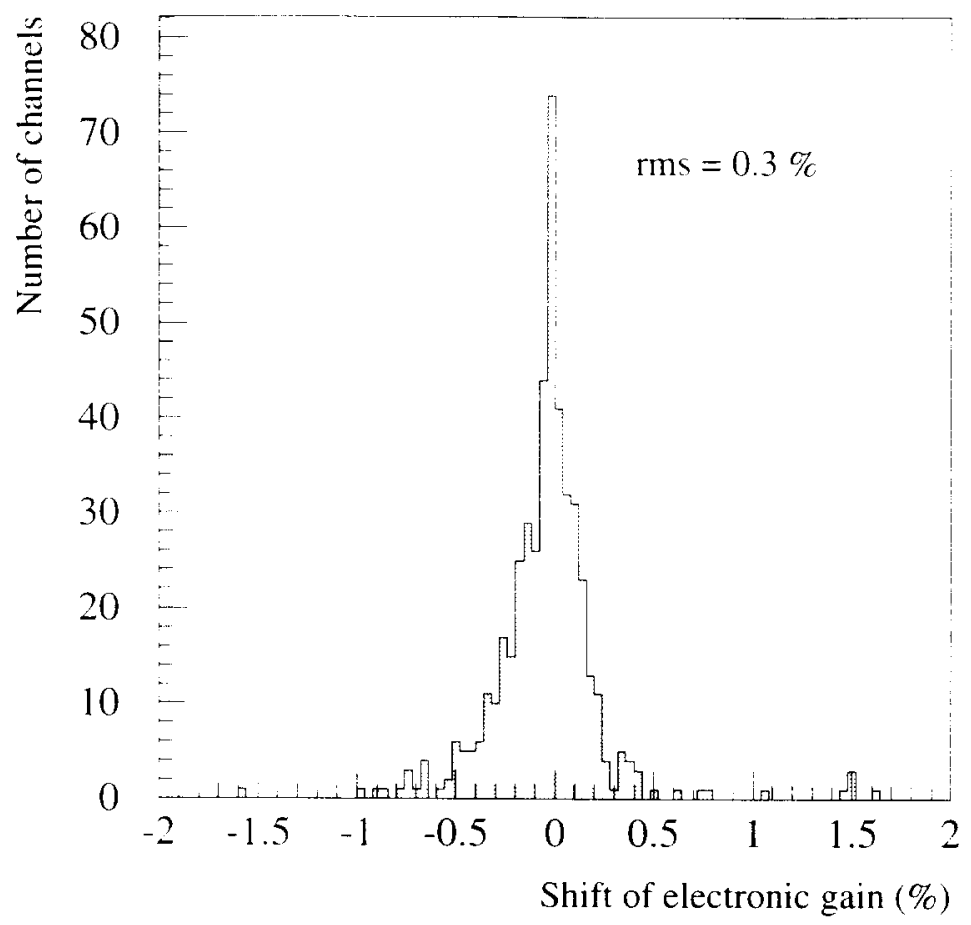

Fig. 4: Two months stability of the electronic chain extracted directly from pulser measurements.

\subsection{Trigger}

The basic function of the BEMC' trigger schematically shown in Fig. 5 is to select the events with localized energy depositions in the BEMC stacks. For this purpose the lowest granularity. corresponding to individual stacks. is used for building the trigger quantities.

Analog signals from photodiodes connected to long WLS in one stack (usually four) are first summed up to form the stack response. The electrical signals differ due to the light properties of the various stack types. The difference is up to a factor 2.5 for the triangularly shaped stack: with respect to the square types. Therefore 88 stack responses are equalized to the same encrgy scale using the known calibration factors translated into adjustable resistor values in the G.AIN card. This set of variable resistors is also used to compensate for malfunctioning individual stack channels. The unique assignment of the energy depositions in all stacks to a single HERA bunch crossing is possible with the time response of the BEMC front-end electronics (photodiodes and preamplifiers). In order to assure minimal timing spread of the individual stack signals adjustable delays are used.

The stack energy signal is further processed in an amplitude and time discriminator (ATD) module. The ATD shaper consist of three parts. An edge extractor sums the direct analog signal with the same inverted and delayed one. A 120 ns delay ensures that the leading edge of the input signal is preserved on the output of this edge extractor. The falling edge of the output signal is $300 \mathrm{~ns}$ long. This signal is afterwards differentiated and a bipolar pulse is created. The peak in the output signal of the edge extractor, corresponding to a $t_{0}$, is determined by a zero crossing detector using the differentiated signal. The output signal of the differentiator is again integrated to obtain good energy resolution.

Two sets of thresholds that are controlled and monitored by threshold modules (THR.II) are applied to the stack signals. A low stack threshold (LT) is used to suppress the noise contribution to the cluster energies and to the total BEMC energy and a high stack threshold 


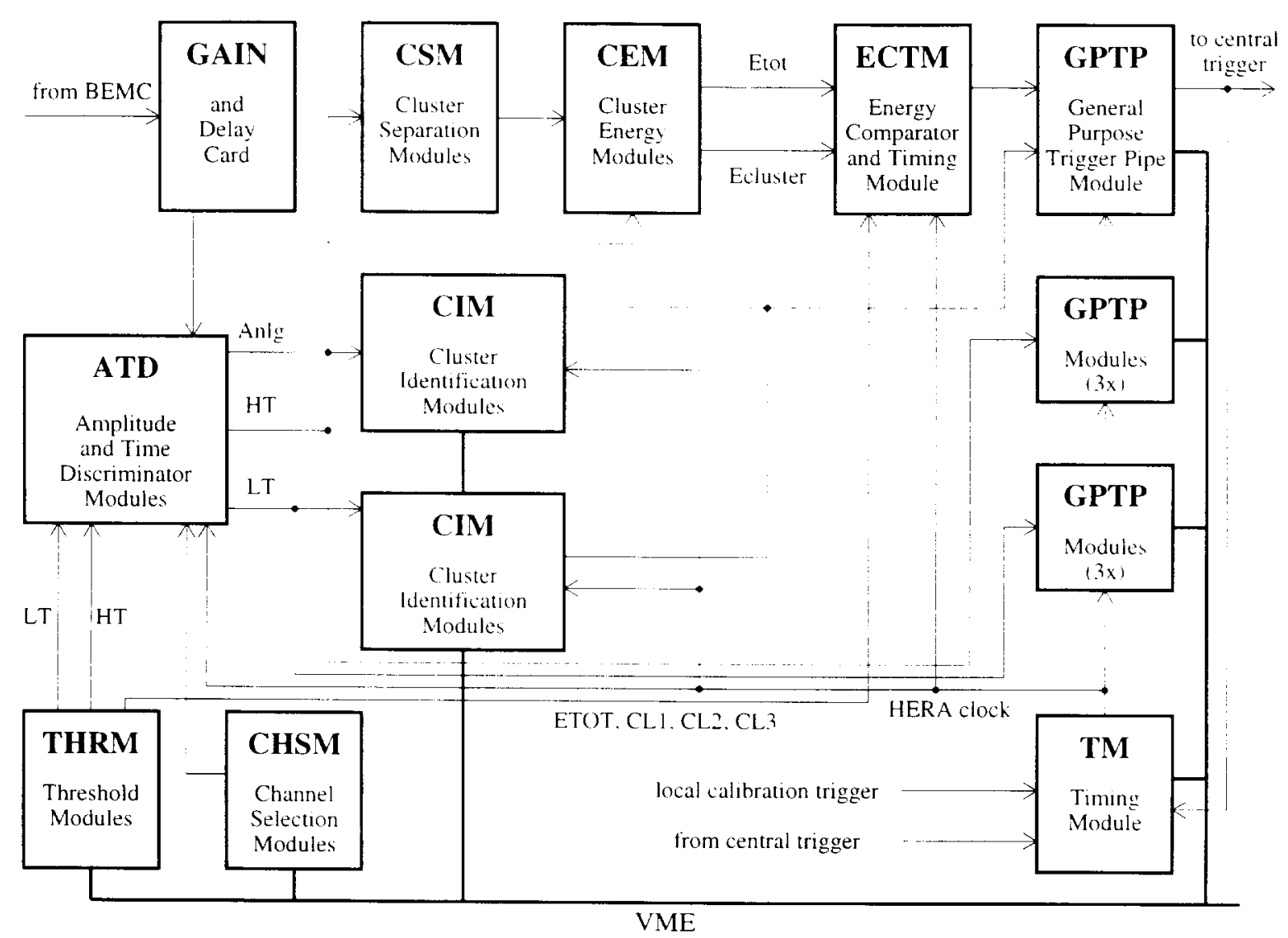

Fig. 5: Schematic layout of the BEMc' trigger.

(cluster seed threshold - HT) serven as a cluster initiator for the cluster separation module (CSM). Typical values of 1.3 GeV for the low and 2.3 GeV for the high threshold are used. For the determination of noise thresholds a threshold ramp procedure based on counting of the noise crents within a specified tine interval was used. The cluster seed threshold values are the result of a compromise between the cluster selectivity and the trigger inefficiencies due to the spreat of the electromagnetir shower into seseral stacks at the stack boundaries. Stack responses can be individually disabled or enabled ria channel selection modules (CHSM).

Afterwards the trigger logic in separated into two independent branches:

- the analog branch (Anlg) which forms analog sums (cluster and the total energies) and

- the digital branch which is based on 2 x kn stack threshold bit patterns for topology and multiplicity determination for the low (LT) and high (HT) thresholds.

Predefined hardwired (luster topologies based on simulation studies of the electron response in the BEMC are put into the cluster separation module (CSM). which detects the cluster seeds and assigns neighbouring stackis above the noise theshold to form trigger clusters. The corresponding energies are summed in the cluster energy module (CEM). In the energy comparaton and timing module (E('T.VI the analog sum of all clusters (Ecluster) is compared 10 cluster thresholds to form three cluster trigger elements labeled CL1. CL2 and CL3 corresponding to different energy thresholds. The toral energy summed over all stacks exceeding the low threntold (Etot) is compared to a threshold forming the total energy element (ETOT). In parallel the cluster energy and the total energy sums are digitized by 6-bit FADCs to be used with other trigger elements in the central trigger logic. 
The general concept over the last three years luminosity periods was to set the highest threshold (CL3) for fully efficient triggering of the events with quasi-elastic scattered electrons (high-x region, kinematic peak) to be used mostly for BEMC calibration and monitoring of the ToF scintillator efficiencies. The medium threshold ( ' $\mathrm{L} 2)$ was used together with a timing veto from the ToF as the standard DIS trigger of low $Q^{2}$ events. The lowest one (CL1) was employed in coincidences with track trigger elements to reach the very low-x region of the neutral current deep inelastic scattering.

The programmable look up tables of the cluster identification modules (CIM) based on the $\mathrm{x}$ and $\mathrm{y}$ projections of the low (IT) and high (HT) stack threshold topologies in the BEMC' provide cluster multiplicity trigger elements (one or more clusters. exactly one cluster).

A trigger interface module (timing module TM) allows switching of the control signals between the $\mathrm{H} 1$ central trigger logic (for the standard data taking mode) and the local pulser (for the stand-alone adjustments and checks) via VME control. In total 189 bits are fed into digital shift registers ( GPTP - (ieneral Purpose Trigger Pipe module [7]) with 32 bunch crossings pipeline depth with VME readout. The position of the nominal bunch crossing in the pipeline can be adjusted using a programmable over-run signal. The readout of the nominal bunch crosuing and two bunch crossing, before and after (in total five) was used to minimize the amount of transferred data. Such information was sufficient to identify possible pile-ups especially in the analog processing of the readout with a longer shaping time. The trigger readout is a vital tool for the understanding and monitoring of the trigger behavior. 16 bit look-up tables allow the building of basic trigger element combinations to be sent to the $\mathrm{H} 1$ central trigger logic. The fast BEMC trigger response is delayed by 12 HERA bunch crossings at the input 10 the $\mathrm{H} 1$ central trigger. This delay is defined by the slowest $\mathrm{H} 1$ trigger elements entering the contral trigger logic.

Already in the first studies of the photodiode calorimeter readout [8]. the sensitivity of the photodiodes to the passage of particles directly through the depletion laver of the photodiond. was recognized. Therefore the necessity of protecting the photodiodes from high backingund particle fluxes was stated. The inportance of this effect (later referred to as the nucluat connum effect) especially for stand-alone calorimeter triggering was realized only with increaned current stored in the HERA accelerator ring. The photodiode sensitivity to gamma rays was verified using a radioactive ${ }^{50}$ Co source illuminating the BEMC front-end electronics (i.e. from the hack of the stacks). The equivalent energy range was found exceeding a level of more than 30 (in) The influence of the most important source of the soft gamma rays - the accelerator inchrotron radiation - was reduced by lead shielding of the beam pipe in the BEMC region.

In order to suppress this type of fake trigger an approach based on the fourfold redundancy of the stack energy information was used (each stack is read out by four photodiodes). Particlen depositing their energy in scintillator material have only a limited range of response sharino between four WLS (photodiodes). which was carefully checked by detailed scanning in the tru beams. Therefore the comparison of energy depositions in the individual photodiodes after meise suppression with a sufficiently high energy threshold was used 10 identify such false triggers. The 16 most affected stacks near the beam pipe were equipped to provide identification of the nuclear counter effect for each bunch crossing. These modules were based on the analog sum. of two photodiodes (to decrease the amonnt of electronics). which were shaped and put into the comparators with manually adjustable thresholds.

An important trigger rate reduction. especially for the stand-alone subtriggers. is provided by the level $t$ of the $\mathrm{H} /$ trigger system [1], which in the case of the BEMC trigger utilizes the possibility of running the complete BEMC reconstruction code to reconstruct individual WI.S energies and to cluster the energy depositions. New inputs are used as a basis for level t trigger decisions with respect to level 1 . Ievel $t$ in especially effective for suppressing the triggers due 
to the nuclear counter effect in lhe stackis not equipped with veto logic. This process is based on the cross check of the individual channel energy depositions in the same stacks using the energy reconstructed from the BEMC analog readout. Threshold sharpening. which includes also trigger noise suppression. is another important rate reduction due to the steeply rising rate distribution for decreasing energy. In addition the events originating from below the BE.MC (the synchrotron mask hit by the tail or displacement of the proton beam) are suppressed on the basis of an approximative vertex algorithm.

\subsection{Slow control and monitoring}

For stable and reliable operation of the calorimeter and the associated trigger a system for stow control and monitoring has been set up. The slow control of the BEMC and its inclusive trigger is a part of the overall HI slow control system [1] and checks the voltages. currents and temperatures of all key electronic parts. In addition a computer controlled stand-alone monitoring system using a MarVEE interface [9] was built to monitor and control the temperature. photodiode bias voltage. photodiode leakage currents and the trigger rates.

In each horizontal row of the BEMC stacks. close to the front-end electronics. temperature probes are installed. The signals from the temperature probes are digitized and read out using a dedicated processor [10]. The actual status is available and recorded into a disk file. The monitoring program compares the actual temperature to preselected limits and in the case of dangerous temperature increase the power supply unit for the front-end electronics is switched off. This action is accompanied by visual and audible alarms. This option was demonstrated to be important for safe operation of the front-end electronics taking into account the tightly packed and enclosed space inside the liquid argon cryostat barrel. shared in addition by all the central and forward trackers together with the ToF scintillator system [1].

The photodiode bias coltages can be set and monitored for each individual stack using a bias voltage supply unit. The photodiode leakage currents for each stack (i.e. the sum of $x$ photodiodes) is displayed and recorded. The leakage current change is dominated by the temperature dependence of the photodiode dark current. which according to [5] increases by a factor 1.15 per $1^{\circ} \mathrm{C}$. For the usual $121^{\circ} \mathrm{C}$ rise of the temperature for the luminosity runs (all power supplies switched on ) w.r.t. periods without luminosity the photodiode dark current increases by a factor 6 . Beside temperature related changes no significant increase of photodiode dark currents was observed.

For the practical operation of the BE.MC' trigger a nonitoring system based on VME programmable trigger rate monitoring modules [11] was built. The number of counts of each low and high stack threshold during the preselected time interval. converted into the corresponding rate. is displaved for each individual stack using a color scale. In addition the rates of all trigger elements sent to the III central trigger logic are available. Such a display provides a very effective tool for fast identification of hardware failures and is important for the understanding of beam and background conditions (asymmetry of the beam position, beam induced background). Predefined relative trigger rate changes of all 184 monitored bits are sent to and recorded in the slow control database.

Another hardware tool fer guick checking of the readout chain including the opt ical components is the BEMC laser monitor ststem. Figure 6 shows the spread of optical responses of all BEMC channels after $1: 3$ and 52 days. 


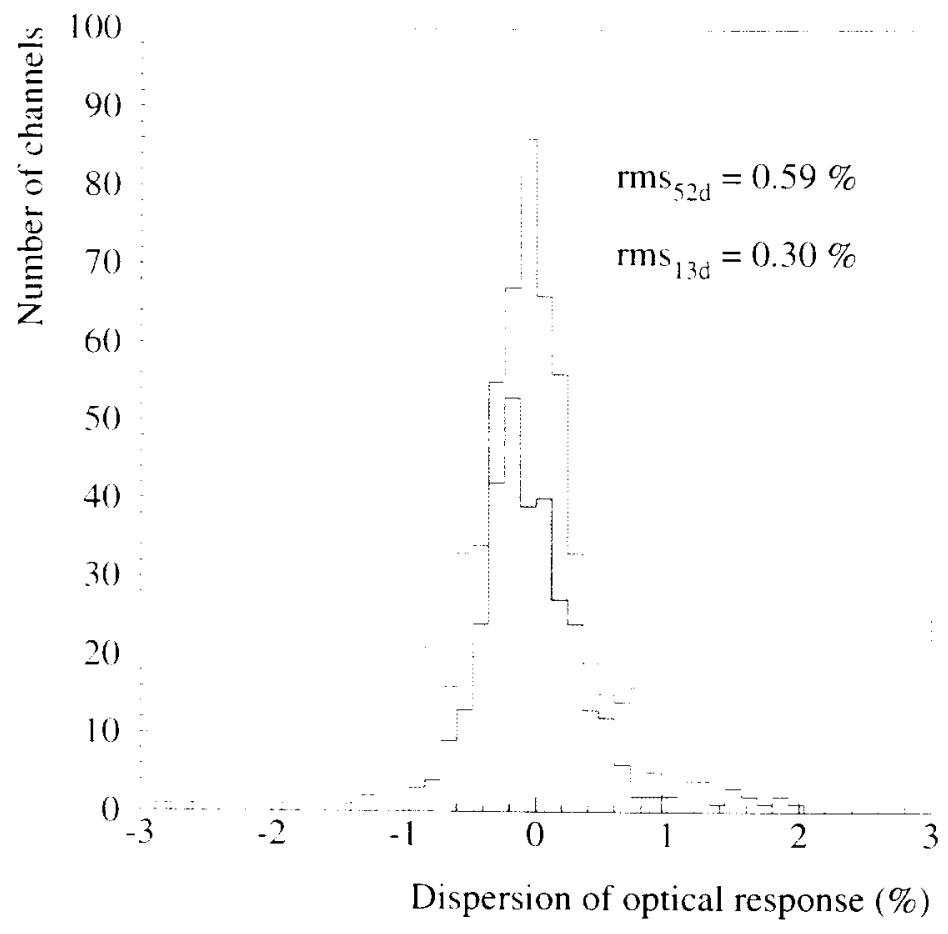

Fig. 6: Spread of optical responses of all BEM( channels after 13 days (shaded) and 52 davs.

\section{Calibration procedure}

For the measurement of structure functions in DIS the determination of the kinematic variables $r$ and $Q^{2}$ using the reconstructed energy and the angle of the scattered electron is recuured [12]. In this case a precise energy calibration of the detector to a $1 \%$ level is crucial [1:3]. The calibration of the BEMC was done in two steps: initial calibration in test beams improved by $r p$ data itself. In the preparation phase a calibration of each single stack was performed using the electron test beam at DESY and for a few stacks also in the CERN SPS. For the transfer of the initial electron energy scale into the $\mathrm{H} 1$ environment the aim was to achieve a precision of calibration of better than $10 \%$. This goal was achieved despite the nontrivial effects of magnetic field. nitrogen atmosphere. scintillator aging. replacement of the test beam electronics by the final ones. a new electronic pulser system and uncertainties of the test beam momentum scale. With the start of hminosity operation the physics data soon allowed one to improve the knowledge of the energy scale. With increased luminosity delivered by the HERA accelerator the design goal of $1 \%$ precision of the global energy calibration for scattered electrons in DIS was accomplished.

\subsection{Initial calibration}

The stack energy was defined as an arithmetic mean of the four channel responses corresponding to the long WLS. In analogy the response of backward part was defined as the arithmetic mean of the two short WLS.

The initial BEMC energy calibration is based on 3 different sources:

- electron test beann data at Dessy.

- electron. pion and muon beam runs at the ('ERN SPS. 


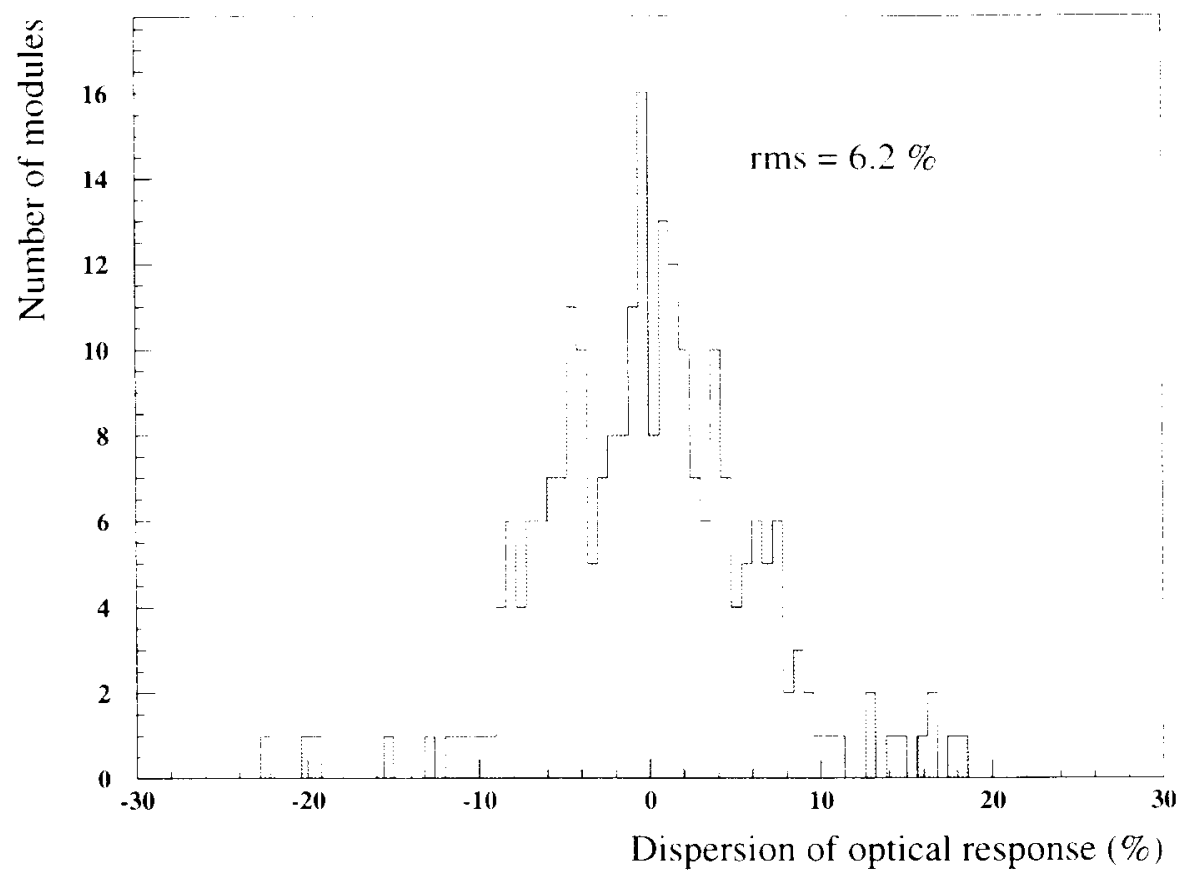

Fig. 7 : Spread of equalization factors for channels with long WLS in square stacks.

- high statistics cosmic muon data.

At DESY all square stackin were calibrated to a $0.5 \%$ level relative to each other using an clectron beam of energy ; (iel. Fig. $T$ shows the spread of calibration factors for all channels with long WLS in square stacks normalized to their average. This figure illustrates the small stack to stack variation in the optical response of square stacks despite all imperfections of optical couplings between the sampling structure. the WLS and the photodiodes.

For the setup phase of $\mathrm{HI}$ the calibration of the non-square stacks was performed using cosmics. Despite the noise of the photodiodes preventing the detection of the cosmic muons on an event-by-event basis. high statistics data triggered by external scintillators allows an identification of the peak positions of the energy distributions. The cross-check of this method with known calibration factors for square stacks indicates very good correlation and therefore the conclusion of $5 \%$ relative calibration precision of all non-square stacks with respect to square can be drawn.

The calibration of channels connected to short WLS is not possible using electron beams since electromagnetic showers are mostly contained in the forward part of each stack leaving only the fluctuating tails in the backward part covered by short WLS. Esing a high energy $\mu$-beam at CERN the ratio of light output from the short and long WLS was normalized to the corresponding ionization losses of muons taking into account the path lengths. Taking into account the spread from Fig. $T$ and considering the non-uniformity in light collection over the ared of one stack for the short WIS one gets a calibration to $20 \%$.

\subsection{In situ calibration}

Three methods described betow were used to reach and to monitor the desired $1 \mathrm{t}$ precision of the electron energy scale:

- kinematic peak mothod. 
- double angle method.

- QED Compton method.

The absolute energy scale for electrons at HERA energy has been fixed using the kinematic peak method. The double angle method provides an independent cross check of the energy scale. The QED Compton method allows one to test the energy scale over the whole energy. range of electrons detected in the BL.MC'.

\subsubsection{Energy calibration using the kinematic peak}

The energy distribution of quasi-elastically scattered electrons in the region close to the beam encrgy is dominated by a pronounced peak (commonly referred to as the kinematic peak). This peak has an asymmetric shape. The shape of the high energy side is dominated by calorimeter resolution and is therefore to good approximation gaussian. whereas the low energy side gets more asymmetric with increasing electron scattering angle and starts to be sensitive to the proton structure function. A minimization method to adjust the observed energy spectra to the simulated ones was developed. Monte Carlo events were generated by the $\epsilon p$ DIS generator D.JANGO [14]. The response of the full $\mathrm{H} 1$ detector was simulated and further reconstructed to provide a large statistics sample of DIS events.

In order to minimize the influence of the proton structure. the low energy cut for the comparison of the simulated and measured data was initially put only a few GeV below the electron beam energy and the sensitivity to the selection of this cut was included in the sistematic error. In addition a very small sensitivity to the input structure function was included in the sistematic error. With improved knowledge of the proton structure function provided by the IIERA experiments the effect of this cut and the selection of input structure function was negligible.

The relatively high cross sections exceeding $100 \mathrm{nb}$ for scattered electrons in the angular acceptance of the BEMC (depending on angular and energy cuts) allowed rapid collection of a high statistics sample and performance of an impact point dependent calibration of the BEM('. During the 1994 luminosity period $\mathrm{H} 1$ collected approximately $4 \mathrm{pb}^{-1}$ with a corresponding sample of kinematic peak events exceeding $\bar{i} \times 10^{5}$. This sample allowed the calibration of individual stacks and the study of the response uniformity for bins of $1 \mathrm{~cm}$ size in the beam pipe region.

\subsubsection{The double angle method}

In the nearly hermetic $\mathrm{HI}$ detector it is possible to measure the scattered electron together with the hadronic final state. Csing the topology of DIS cvents - namely the angles of the scattered electron $\theta_{t}$ and the hadronic final state ("current-jet") $\theta_{j, t}$ it is possible to calculate the energ. of the scattered electron $L_{\text {, }}$ using the following relation:

$$
E^{\prime}=\frac{2 E_{i}}{\sin \theta \cdot\left(\tan \frac{\theta_{c}}{2}+\tan \frac{\left.\theta_{2 \leq t}\right)}{\frac{2}{2}}\right)} .
$$

where $E_{e}$ is the incident electron energy.

The main problem of this method is the reconstruction of the current-jet polar angle $\theta_{j, t}$. ['sing the method of Jacquet-Blondel [15] this angle can be determined:

$$
\cos \theta_{j, 1}=\frac{\sum_{h} E_{h} \cos \theta_{h}}{\sum_{h} F_{h}}
$$


where the summation is made wer all particles $h$ of the hadronic final state with scattering angle $\theta_{h}$ and energy $E_{h}$. Especially for small $\theta_{z}$ the reconstructed angle can be systematically increased due to energy loses in the beam pipe and therefore a safe angular cut should be applied. For kinematic reasons this method "an only achieve a sufficient level of precision in the energy region close to the electron beam energy $E_{\text {. }}$. in which the calculated energy has mininum sensitjvity to the errors of the meanured angles of the current-jet and also the scattered electron.

\subsubsection{QED Compton method}

The decomposition of the QED radiative corrections to neutral-current DIS in the leading logarithmic approximation reveals the part associated with nearly collinear emission of the exchanged photon from quarks or from the proton the quasi-real QED Compton process. This process corresponds to the Compton scattering of a quasi-real photon on an incident electron [16]. The dominant contribution to compton scattering is due to the elastic channel ( $p-(p)$. The small inelastic contribution is largely suppressed by looking at coplanar $\epsilon$ - $;$ events.

The elastic QED compton events have a very clean experimental signature. The final state electron and the photon at large transverse momenta are nearly balanced and accompanied by no hadronic activity. The outgoing electron and photon are emitted mostly into the barkward region where the BEMC is located.

Six kinematic variables describing the QED Compton process are directly measured namely the emission angles of the final state particles $\left(\theta_{c}, \theta_{2}\right)$ and $\left(\theta_{+}, \theta_{2}\right)$ and the corresponding energies $\because \because$ and $E_{\imath}$. Neglecting the transverse momentum of the $(c-\gamma)$ system only three of these variables are independent and the following three conditions constrain the hinenatic variables of the QED) Compton process:

$$
\Delta 0=\pi-|0,-0| \simeq 0
$$

where to is the acoplanarity angle. and

$$
\begin{aligned}
& E_{1}^{\prime}\left(\theta_{,} \theta_{\gamma}\right)=\frac{2 E_{2} \sin \theta_{\gamma}}{\sin \theta_{\tau}+\sin \theta_{\gamma}-\sin \left(\theta_{t}+\theta_{\gamma}\right)} \\
& \therefore\left(\theta_{2} \theta_{2}\right)=\frac{2 E_{2} \sin \theta_{2}}{\sin \theta_{r}+\sin \theta_{2}-\sin \left(\theta_{e}+\theta_{2}\right)} .
\end{aligned}
$$

These strong constraint make this process sery well suited for calibration of the BEMC' [1 $\bar{c}]$ over a large energy range. This is the only process with sufficiently large cross section for the determination of the linearity from e $p$-data itself (see section 4.1.3). The cross section of the elastic QED Compton process is approximately two orders of magnitude smaller than the Born cross section in DIS. Which is a drawback of this method in comparison with both the previous ones.

\subsection{Special effects}

The influence of the axial magnetic field on the light vield was studied. The known variation of relative light yield of scintillator [ $[N] I$ with increased magnetic field $B$ was confirmed and approximated by a linear dependence $I=1+0.053 \times B$ in the range up to 1.5 Tesla. This was used to transport the calibration factors from the test beam with no magnetic field into the III environment. For the magnetic field in the position of the BE:MC this effect leads to an increase of the response of approximately $5 \%$. The effect was verified in special luminosity runs with no magnetic field using the kinematic peak method. 
In the HI detector the BLAC' is placed in a nit rogen atmosphere used for safety reacons due 10 the flanmable gases of the tracker system. Therefore the effect of the nitrogen replacing the air during the test bean calibrations on the scintillator light yeld was also investigated. The effect was studied using a dedicated setup. Replacing the vacuum by nitrogen the reversible decrease of relative scintillation light vield $I$ stabilizing after 90 minutes was found to depend linearly on pressure $p$ of nitrogen: $I=1-0.0025 \times p$ for $p<2$ bar. A more significant response reduction was determined by replacing the vacuum by oxygen: $l=1-0.057 \times p$ for $p<2$ bar. Therefore taking into account the composition of normal air with respect to nitrogen atmosphere a scintillation light yield increase can be expected. The nitrogen concentration at the location of the scintillators is not exactly known and therefore no attempt to correct the effect was made. Due to the stable monitored nitrogen atmosphere [1] inside the hemetically closed volume this effect was taken into account by the above mentioned in situ calibration methods.

$12 \%$ relative decrease of the wo bottom WLs with respect to the top ones was observed for stacks with "top-bottom" orientation (Fig. 2a) of long WLS. causing a sistematic shift of the shower center of gravity reconstructed on the basis of the four WLS responses. This effect is understood to be the consequence of the stack weight compressing the nylon threads. In consequence the gap between scintillator plates and WLS bars got smaller causing a slight optical deterioration of the scintillating light collection. This gravity effect was taken into account offline by $2 \%$ increase of bottom WLS responses.

Due to scintillator aging and possible radiation damage a time dependence of the calibration can be expected. Relative comparisons of kinematic peak positions confirm the time stability to be better than $0.5 \%$ during 6 months of the 1994 run period.

\section{Detector and trigger performance}

\subsection{Response to electrons}

\subsubsection{Energy scale}

In Fig. 8 a comparison of the measured energy distribution of the leading cluster with a simulated one (shaded area) using the structure function GRV [19] is shown after adjustment of the calibration for each individual stack using the kinematic peak method. Good matching of the data and simulation proves the quality of the BEM( calibration and understanding of the detector response. In the 1994 luminosity period the global energy scale for electrons is DIS was adjusted using the kinematic peak method with a precision corresponding to 1 t doninated by sistematic errors.

The calibration was cross checked using the double angle method. The quality of the global scale adjustment is illustrated in Fig. 9. Where the distribution of the relative differences of the clectron energies $E_{20}$ calculated using formula $(1)$ and the measured ones $E_{\text {mea }}$ are shown. The mean of the gaussian distribution was found to be centered at 0 within $0.1 \%$. The width of $4.0 \%$ reflects the broader selected energy and jet intervals. The shaded area in Fig. 9 is the same distribution from simulations using the MRSH structure function [20]. Again the mean was found to be centered at 0 within $0.1 \%$ with a corresponding width of $3.6 \%$. which demonstrates the good understanding of the BEMC energy response including nonuniformity effects.

Detailed studies were made to deremine the angular dependence of the energy calibration using the double angle method. The resulting mean values of the comparison of the calculated electron energy $E_{2,}$ and the measured one $t_{m, n}$ are shown in Fig. 10 as a function of mare $\left(x_{B P C}|\cdot| y_{B P C} \mid\right)$. The quantity max $\left(x_{B P C}|\cdot| y_{B P C} \mid\right)$ is the maximum of the absolute values 


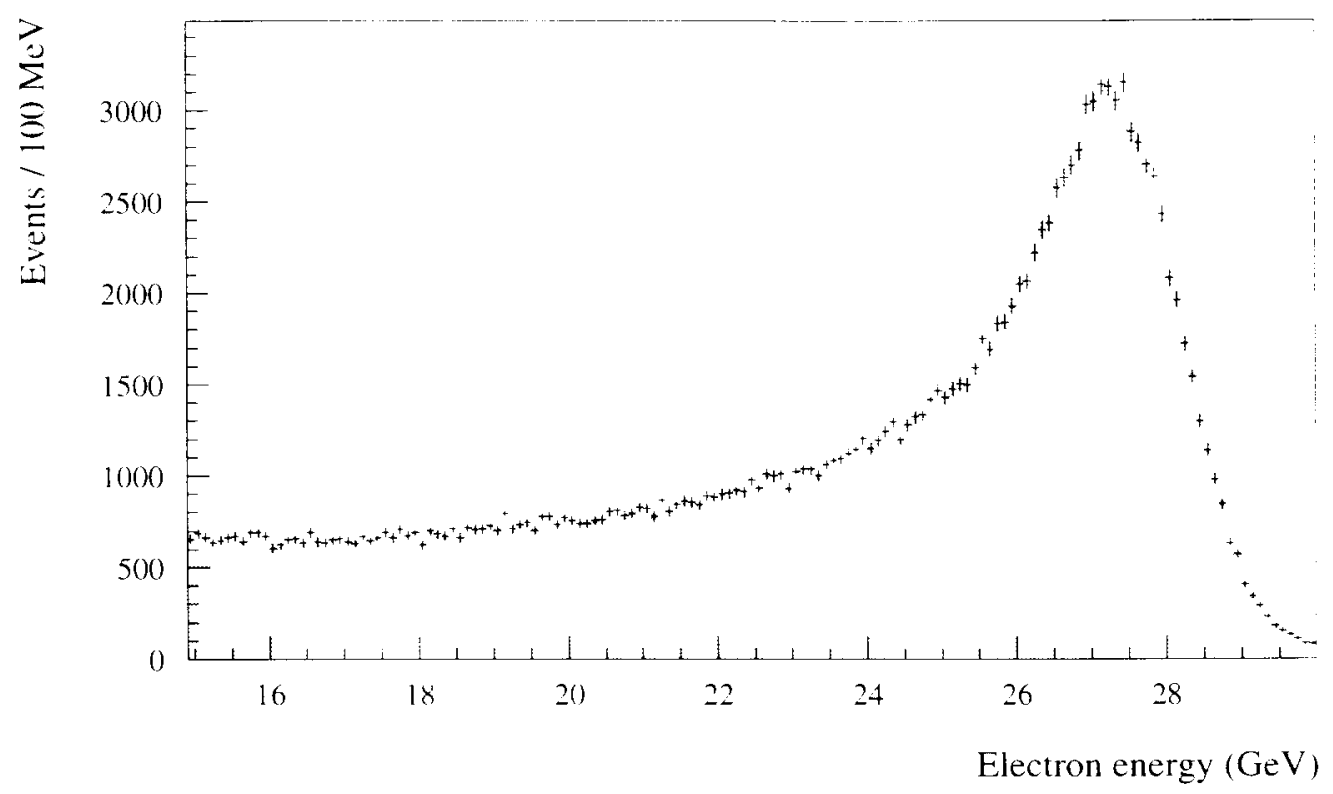

Fig. 8: Energy spectrum of low $Q^{2}$ DIS electrons scattered in the region of square stacks. For simulation (shaded area) the structure function GRV is used.

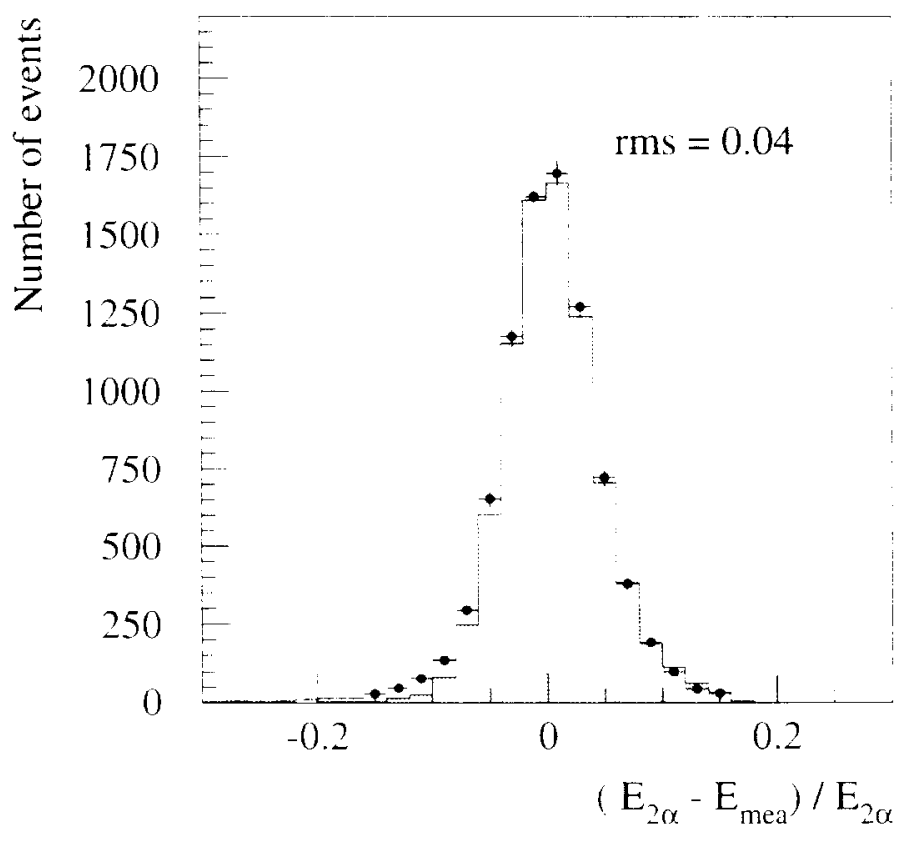

Fig. 9: Adjustment of the energy scale using the double angle method for low $Q^{2}$ DIS events. The shaded area is Monte (arlo with MRSH structure function whereas points represent data.

of the $x$ and $y$ coordinates of the ansociated BPC hit to the electron cluster. reflecting the square topology of the BEMC. These studies proved the energy scale to be known to better than $1 \%$ in the angular range $160^{\circ}<\theta<176^{\circ}$ corresponding approximately to the 32 stacks close to the beam pipe. Outside this region the available statistics do not allow one to reach the sane level of precision. A level of $2 \%$ precision of the energy calibration was reached in the angular region $15.5^{\circ}<\theta<160^{\circ}$ covering approximately the neighbouring square stacks of the above mentioned 32 stacks [21]. Dedicated studies improved the energy scale knowledge in the overlap region between the BEMC and the BBE. corresponding to the angular range $1.50^{\circ}<\theta<1.55^{\circ}$. and decreased the energy seale uncertainties below $5 \%$ in that region [12]. 


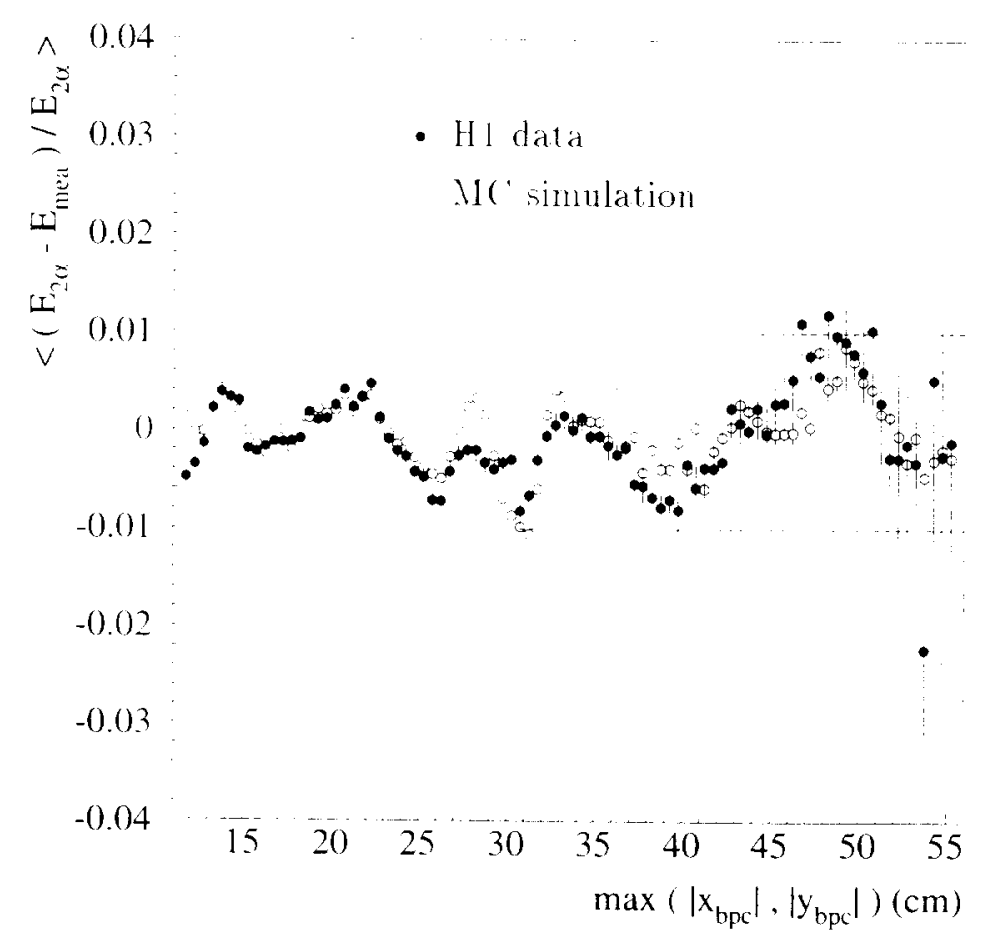

Fig. 10: Distribution of the mean values of the differences between calculated $E_{2,2}$ and measured energy $E_{m \in a}$ of the electron candidate in data and Monte Carlo simulations. The quantity $\max \left(\left|x_{B P C}\right| \cdot\left|y_{B P C}\right|\right)$ is the maximum of the absolute values of the $x$ and $y$ coordinates of the associated $\mathrm{BPC}$ ' hit to the electron cluster.

C'sing the QED Compton "vents the global BEMC energy scale was chected. For the BEXIC angular region the sum of the cluster energies corresponding to electron and photon is shown in Fig. 11a. In the low energy range the inelastic contribution is visible. The constrained fit for 1994 luminosity period data $\left(E_{b, a m}=27.52\right.$ (ieV) reveals a mean position 27.5) (iel with a statistical error $0.3 \%$. The width 1.1 Gel corresponds to a two-cluster convolution of the BEMC resolution.

\subsubsection{Energy resolution}

The energy resolution has been measured at ('ERN and DESY test beams (Fig. 12). Three terms determine the chatacteristicn of the energy resolution. The readout by photodiodes and preamplifiers leads to a constant noise term. This noise has been measured to be 9:3 .leV. Sampling fluctuations are proportional $10 \sqrt{E}$. The limited depth of the stacks leads to leakiage and contributes to the resolution with a term proportional to $E$. A fit of these three terms for the measured points gives the resolution function:

$$
\frac{\sigma}{E}=\frac{0.09-(6)}{F} \therefore \frac{0.100(3)}{\sqrt{E}} \div 0.010(3) \quad(E \text { in } \mathrm{GeV})
$$

In the $\mathrm{H} 1$ enviromment the energy resolution is not directly measurable. It is possible 1.0 extract the information indirectly from the high energy side of the kinematic peak. Which is dominated by calorimeter resolution. The above resolution function was adopted for the BE.MC calorimeter in the $\mathrm{H} l$ environment taking into account two main differences: the energs of a scattered electron is reconstructed from the most energetic stach and its eight neighbours. This increases the noise term by a factor 3 with respect to a single stack. In addition the change of the 

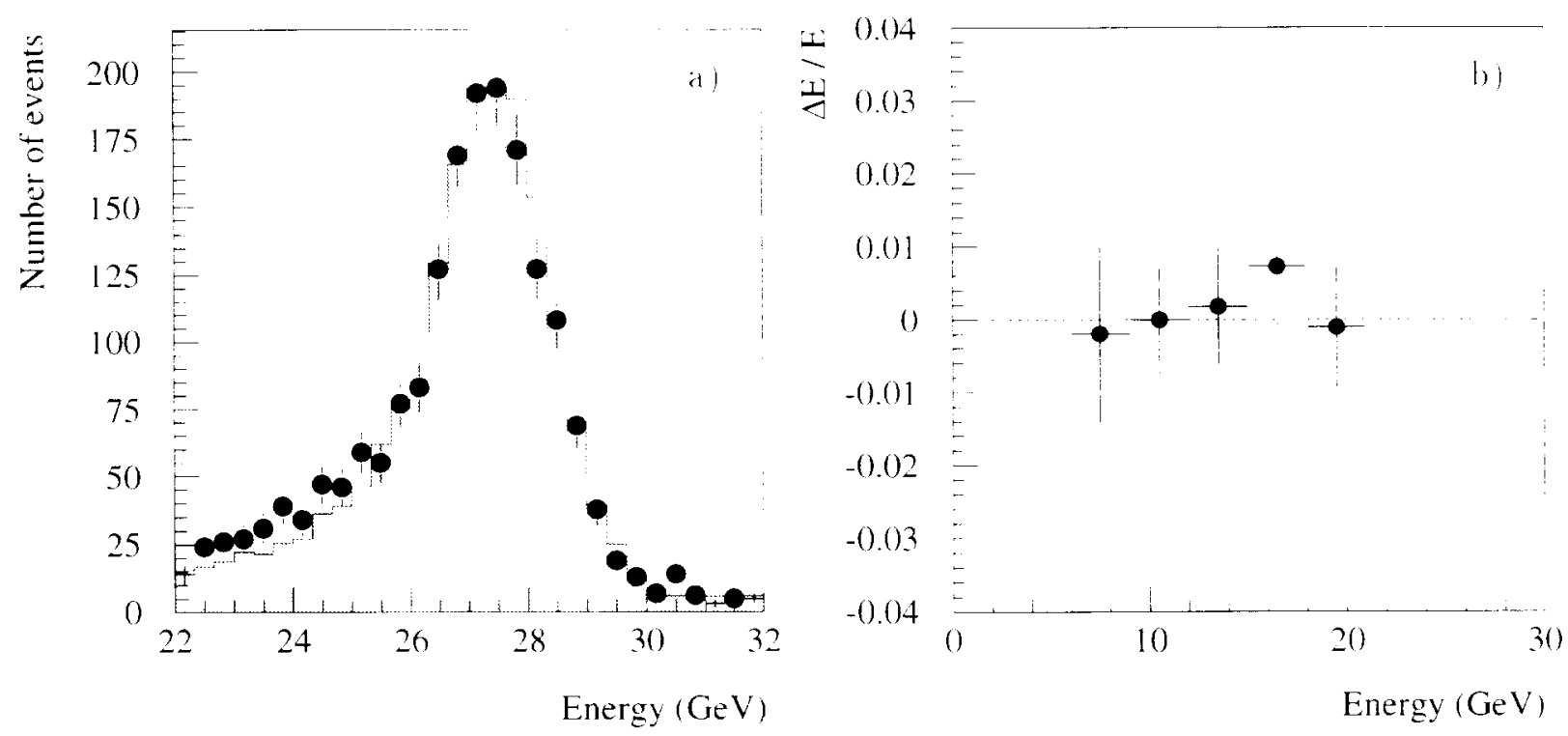

Fig. 11: a) Energy sum of scattered electron and photon in QED Compton erents compared to XI' simulation (shaded). The peak corresponds to 199-t luminosity period electron beam energy of $27.5 \mathrm{GeV}$. b) The linearity of the BEMC determined with QED Compton events is better than $1 \%$.

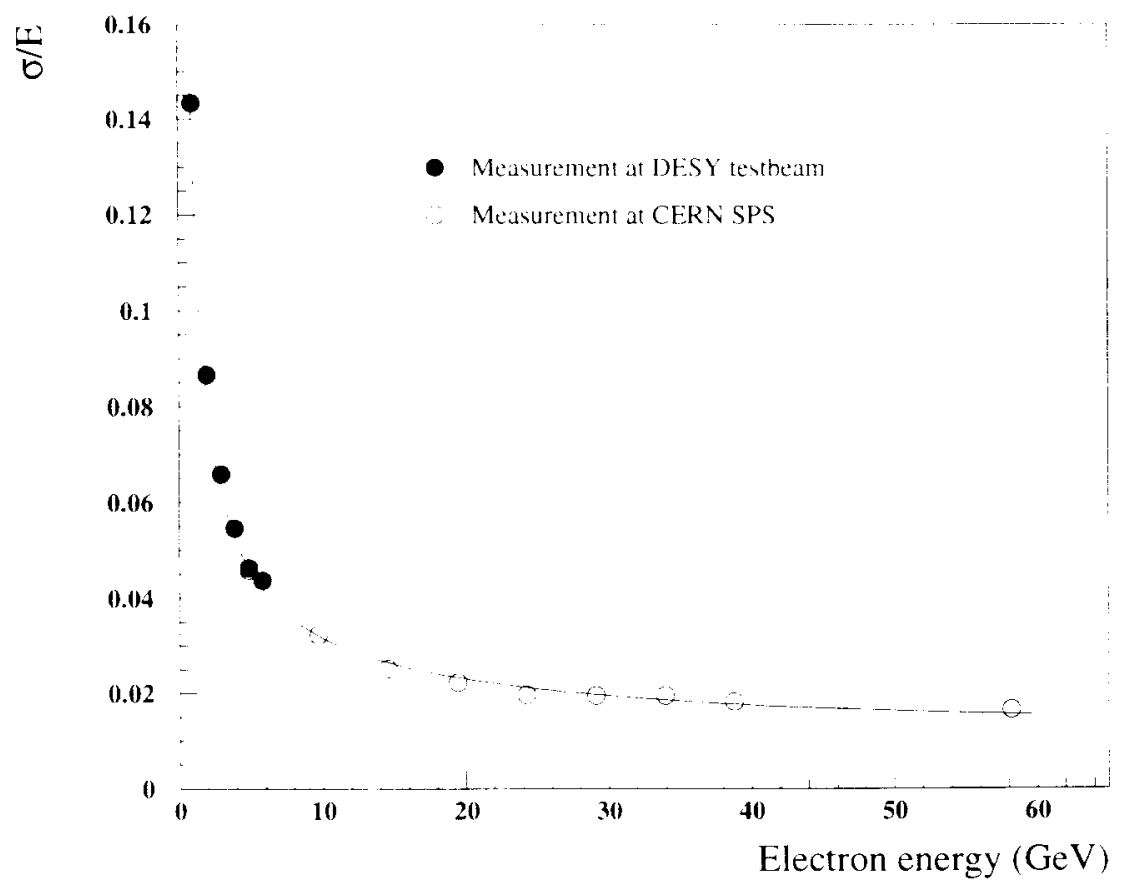

Fig. 12: Energy resolution of a single square stack. The measurements were made at the DESY test beam for electron energies in the range from 1 to 6 (iel and at the (ERN SPS for energies from 5 to $60 \mathrm{CeV}$.

shaping time constant to the find one slightly increased the noise of a single stack. Therefore the noise contribution tern is $390 \mathrm{MeV}$. Since the sampling structure did not change the sampling term is fixed to $100 \mathrm{MeV}^{1 / 2}$. Stack-to-stack variations of the calibration. which were minimized by individual stack calibration. and inhomogeneities of the energy response especially near the stack borders deteriorate the resolution and increase the constant term. Adjustment of the kinematic peak width for simulated electron response to that for data gives a value of $1 . \bar{c} \%$. 
The BEMC resolution function in then given by:

$$
\frac{\sigma}{E}=\frac{0.39(2)}{E} \div \frac{0.100(3)}{\sqrt{E}}-0.017(5) \quad(E \text { in (iel) }
$$

\subsubsection{Linearity}

At CERN the response linearity of a few BEMC stactis was verified using different electron beam energies and found to be better than $1 \%$ in the range from 5 to 30 GeV. The QED Compton events were uned to verify the response linearity of the BEMC in the $\mathrm{H} 1$ detector. (OI the basis of the cluster positions the expected energies can be calculated using relations ( $t$ ). A gaussian fit of the difference between calculated and measured cluster energies in five selected energy bins reveals the mean positions shown in Fig. 1/b. The vertical error bars indicate the errors of the mean position fit and the systematic error of the polar angle reconstruction whereas horizontal ones are the energy bin widths. This data confirms the linearity of the BFMC electromagnetic scale to within $1 \%$ in the range from approximately 5 to $22 \mathrm{GeV}$ (restricted by encrgy requirements applied to non-leading cluster).

\subsubsection{Uniformity}

A large effort was spent to prepare optimal shadowing structures (grey patterns) on the paper sheets inserted between each lead and scintillator plate to homogenize the response over the stack surface area. The optimal patterns for the different stack types were determined by optical scanning of one scintillator plate coupled to WLS and readout electronics. using a point light source. Applying shadowing structures the resulting homogeneity was proven to be beter than $1 \%$ for point light sources.

The amount of dead material in front of the BEAIC represents about $1 X_{0}$, which locally increases up to $2 X_{0}$. In addition energy losses occur if the shower development takes place with a significant fraction between the stackis in cracks with WLS bars. These losses extend up to $8 \%$. The corrections for the decrease of response due to cracks are based on detailed scanning using Monte Carlo simulations. A large effort was spent to tune the simulation of the calorimeter response to the test data and to describe properly all important materials before the calorimeter. Using correction tables the relative energy loss in crack regions can be corrected defining the entry point into BEMC by the BPC. Analysis allowed a local event-by-event correction which sharpened the kinematic peak.

I'sing the double angle method the resulting non uniformities including all additional external sources of inhomogeneities are found to be less than $1 \%$ after applying crack corrections in the angular region $160^{\circ}<\theta<176^{\circ}$ (Fig. 10). The residual nonuniformities of the calorimeter response are well described by simulation.

\subsubsection{Space point reconstruction}

Since the Molière radius of BLAC stacks is $\approx 3.4 \mathrm{~cm}$. an electromagnetic shower is quite often absorbed mainly in one stack. Therefore the shower center of gravity calculated only from the sharing of energy among different stacks of a cluster is very inaccurate. The energy of each stack is reconstructed from the light collection of the four photodiodes, which. due to light attenuation. depend strongly on the position of the shower inside that stack. The dependence of the light collection on the impact point of an electron has been studied and parameterized using a set of appropriate functions. 
The energy weighted arrage of the energy depositions in each stack of a cluster define: the center of gravity of this chuster. The space point resolution one can get with this method is shown in Fig. 13. The entry point of an electron into the BEMC is taken from the BPC. From all good electron candidates above 10 (iel with a misidentification probability below $1 \%$ only those with exactly I BP( hit were selected to suppress the effect of preshowering which introduces additional smearing for the determination of the electron entrance point. For these events the space resolution determined by the BP(' is better than $1.5 \mathrm{~mm}$ - much beter than for the BEMC:

In a coordinate system with the BP( hit in its center the distribution of the associated chuster defines the space point resolution which is $i$ mm including the above mentioned space resolution of the BPC. This value corresponds to a resolution in $\theta$ of approximately 5 mrat.

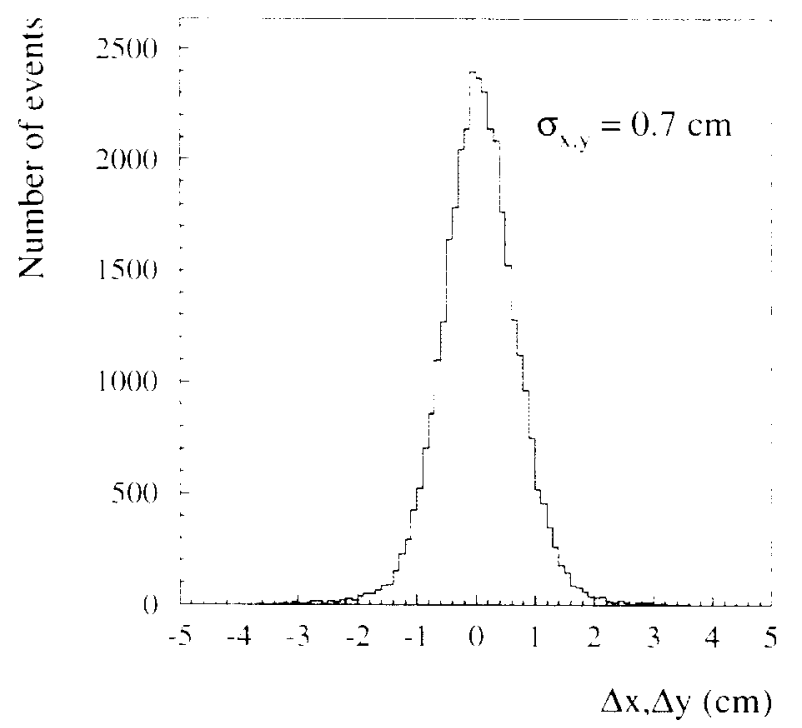

Fig. 13: Space point resolution determined from data for electrons scattered into the BEMC . Shown is the distance between the reconstructed position of a cluster at the BPC plane and corresponding BPC hit for electron energies greater than 10 (iel.

\subsection{Response to hadronic particles and electron identification}

The depth of the BEMc is approximately 1 nuclear interaction length $\lambda$. A distinction between electrons and hadrons in deep inelastic physies plays an important role in the suppression of the main background. i.e. fake scattered electrons. The dominant origins of falsely identified electrons are:

- overlap of charged pions and gammas.

- interacting charged pions.

- $\pi^{0}-\gamma \gamma$ with converting gammas.

The main source of this type of background to electrons is photoproduction which exceeds. by few orders of magnitude. the DIS cross section in the kinematic range corresponding to the BEMC acceptance. In addition. at low energy and low four momentum transfer. both the scattered electron and the curren-jet enter the backward calorimeter. In the low energy region semileptonic heary quark decays are an additional source of real electrons. 


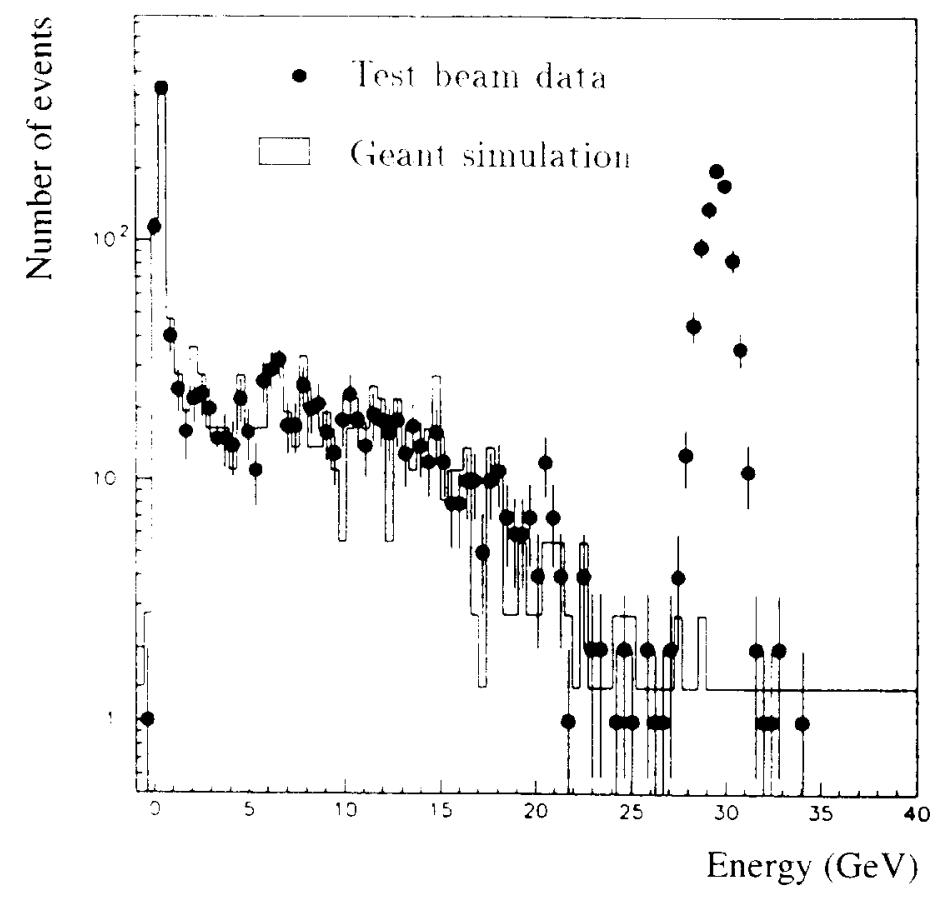

Fig. 14: Spectrum of a 30 (iel mixed positron/pion beam. The measurement was taken at the ('ERNSPS. Data are compared with a detailed detector simulation of charged pions based on the (iEANT package normalized at the signal of minimal ionizing particles. The positron contribution is visible as a peak at 30 (iel.

In Fig. 14 the response of the BEN( to 30 Gel pions is shown. The data was measured at the CERN SPS and is compared to a detailed Monte Carlo simulation based on the GEANI package [22]. The simulation is nomalized to data at the minimal ionizing particle response peak. $40 \%$ of the charged pions traverse the BEMC as minimal ionizing particles. In an average shower pions deposit $\approx 15 \%$ of their energy in the BEMC. The good agreement between the test beam data and the shower simulation justifies the use of the BEMC in measurements of the backward hadronic energy flow.

A tool to suppress the background due to the first and second sources of fake electrons are the quantities based on the lateral shower profile. which is significantly larger for hadronic showers. Electrons produce localized showers concentrated in one or two stacks in contrast to hadrons which produce showers extending over several stacks. Even more in contrast to the scattered electron in DIS. in photoproduction several particles enter the calorimeter in close angular proximity, causing additional "broadening" of the lateral shower profile. In Fig. 15 a quantity called "energy weighted cluster radius" from $\mathrm{Hl}$ data is compared with several Monte Carlo simulations. This quantity is defined:

$$
R=\frac{1}{E} \sum_{i=1}^{n} E_{i} \times \mid r_{i}-r_{i}
$$

where $E$ is the cluster energy. $r$. the cluster center of gravity. $E_{i}, r_{i}$ the energy and center of gravity of stacks forming cluster. The summation is done over all stacks of the cluster. $R$ is small for real electrons. but becomes larger for photoproduction events. For simulation of DIS events the generator DJANCiO [14] is used. Photoproduction is simulated with a generator containing "soft" and "hard" contributions according to [2:3]. The agreement between Monte ('arlo simulation and real data is good. Together with other selection criteria (e.g. matching of the cluster shower center of gravity with the reconstructed BPC point) the quantity $R$ was effectively used to suppress the main background to DIS the fake electrons stemming from photoproduction [12]. 


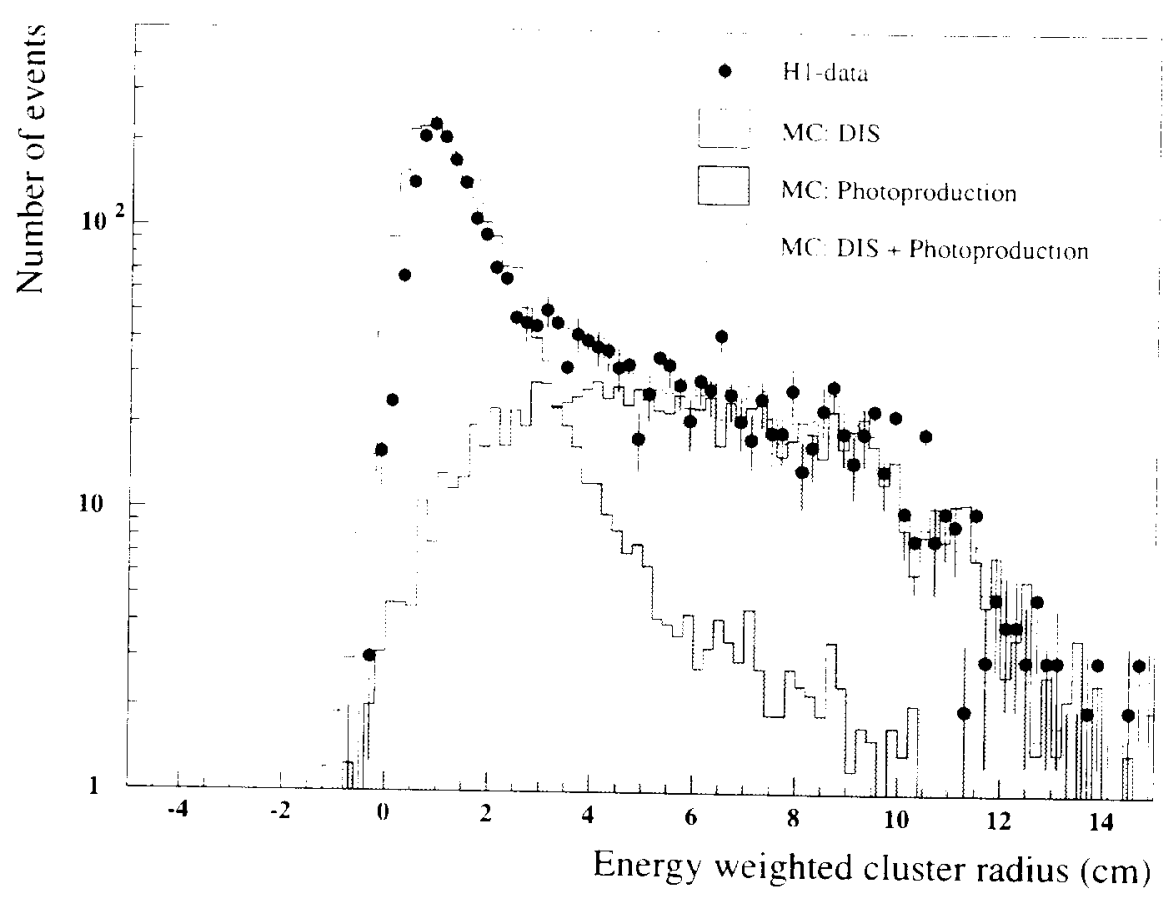

Fig. 15: Distribution of the energy weighted cluster radius of $H 1$ data compared with simulations normalized to the absolute luminosity.

\subsection{Trigger performance}

The efficiency of the BEMC trigger was determined using a sample of events triggered by independent triggers (licpuid argon calorimeter trigger. z-vertex trigger and their coincidences [1]) and the analog readout of the BEMC calorimeter for the determination of the electron energy. Fig. 16 shows the efficiency of the two lower thresholds labeled CL1 and CL2 used to trigger the low-x, low- $Q^{2}$ physich sample. namely ('L2 as the stand-alone calorimeter trigger and CI. in coincidence with tracker triggers. The $50 \%$ trigger efficiencies (frequently referred as thresh olds) for the cluster thresholds ('I. 1 and CI.2 were set to 4.3 GeV and 7.7 GeV respectively. The corresponding widths of these thresholds $(1 \sigma$. determined from the derivative of the threshold efficiency shown in Fig. 16). dominated by the individual stack noise contributions. are 1.0 and 1.1 (ieV respectively. The highest energy threshold ( 13 which is used for triggering of linematic peak events and for monitoring of the ToF efficiency was set to $16.9 \mathrm{GeV}$ with a corresponding threshold width of 1.6 (iel:

The thresholds were set on the basis of rate contributions to the L1 trigger [1]. Two main source of undesired triggers were identified. The most difficult one was caused by a synchrotron mask just below the BLAC $[1]$. Protons scraped off by this mask were the source of the backiground not vetoed by the Tol system installed behind the BENC. These events had very high track multiplicities in the central and forward trackers causing a large dead time due to the tracker readout. In the BEAC these events were triggered mainly by the triangular stacks near the beam pipe. Therefore except for short luminosity periods with stable beam conditions the high stack thresholds of the four innermont triangular stacks were disabled to prevent cluster seeds originating from the beam pipe region. The usual global rate suppression factor due to this was approximately 1.5

Another source of false triggers was due to the nuclear counter effect caused by the pastage of charged particles or gammas directly through the photodiodes. In the HERA environment the 3 main sources of these spurions triggers were identified as: 


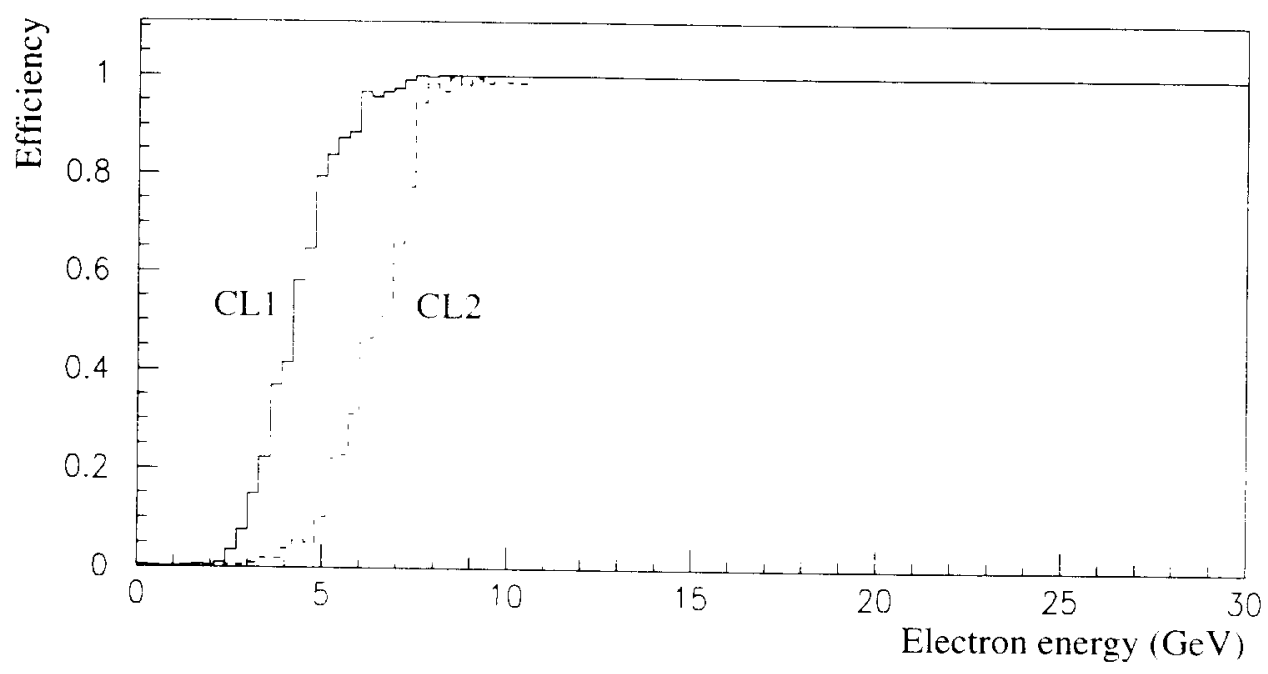

Fig. 16: Trigger efficiency for thresholds ( $\mathrm{L} / \mathrm{l}$ and ('L2

- charged particle flux originating from the proton induced background (beam-gas and beamwall interaction ):

- direct and scattered stuchrotron radiation:

- leaking shower particles originating from the interaction point. most important for phisics analysis.

The dominant contribution responsible for the increase of the trigger rates was the sensitivity of photodiodes to synchrot ron radiation. since triggers due to charged particles originating from proton-gas and proton-wall collisions were effectively vetoed by the ToF system. Lead shielding of the beam pipe region below the BEMC was not sufficient to suppress these triggers. Therefore the 16 innermost stacks (where more than $90 \%$ of these type of fake triggers originate) were equipped with veto electronics (see section 2.3). This veto was applied only for the medium 1hreshold CL2. but not for the lowest threshold ('L 1 for which the coincidence with tracker 1.riggers effectively suppressed these triggers already. The average rate suppression for the standalone calorimeter trigger ('I.2 due to this vetoing exceeded a factor 2.

Depending on the beam currents and conditions the rates of the stand-alone calorimeter trigger CL2 were in the range from $51012 \mathrm{~Hz}$. For average 1994 instantaneous luminosity of about $1.4 \times 10^{30} \mathrm{~cm}^{-2} \mathrm{~s}^{-1}$ the contribution of genuine DIS events was less than $0.5 \mathrm{llz}$. Hore than one order of magnitude observed rate increase was caused by background not retoed by the Tol veto. by the nuclear connter effect due to sinchetron radiation and by photoproduction evellts.

\section{Conclusion}

The desired $1 \%$ global energy calibration of the BEMC for the measurement of scattered electrons in DIS was achieved using an initial test beam calibration improved by in situ calibration methods. Electromagnetic showers can be localized with an accuracy exceeding $\sigma=7 \mathrm{~mm}$ using the distribution of scintillation light into the four WIS. The reliable analog trigger achieved full efficiency for electron energies above 10 GeV using the medium cluster threshold. This trigger was operated with only the ToF veto to collect the most luminosity. The trigger allowed a unicue bunch assignment of the event origin. 
During the three-vears period the successful and stable operation of the BEMC and its associated trigger have allowed the collection of data samples which were the essential ingredients of a large number of physics studies: determination of the proton structure function [12]. observation of diffraction in IDIS [24]. determination of the gluon density [25] and jet analysis in the low $x$ regime $[26]$ to name just a few of them. The experience gained during this time has shown that it is a simple and reliable apparatus with stable characteristics.

\section{Acknowledgements}

It is a pleasure to thank the $\mathrm{H} 1$ collaboration for stimulating motivation and constant interest. We acknowledge the excellent work of the mechanical and electronic workshops of the I. Institut für Experimentalphysik. Cniversität Hamburg and DESY. This work was supported by Bundesministerium für lorschnng und Technologie. FRG under contract numbers 6H H3 3 P and 6HD2-I. by Deutsche Forschungsgemeinschaft. by (i.) C'R grant no. 202/93/242:3 and (i.) 11 ('R grant no. 19095. We also wish to thank the DESY directorate for the hospitality extended to non-DESY members.

\section{References}

[1] HI Collaboration (I. Ab1 t al.). I)ESY 93-103. Hamburg 1993

[2] H1 Calorimeter Group (B. Andrieu. et al.). Nucl. Instr. and Meth. A36 (1993) 460.

[3] Manufactured by hyowa (ias ('hemical Industry.

sets T. Kanon et al. Vucl. Instr, and Meth. $213(1983) 261$.

[4] BEMC group publication in preparation.

[5] Hamamatsu. Technical Data Sheet No. S-504-01.

[6] Floeth Electronic. Landsberg a. Lech. Ciermany

[7] H. T. Duhme et al.. Hl interual note. September 1991.

[8] ( . Blanar et al.. Nucl. Instr and Meth $203(1982) 213$

[9] B. C. Taylor. EC P' Division. ('ERN (iencva 1990)

[10] R. van Staa, H1 internal note HLLSTE( g)-5. Cniversity of Hamburg. 1990

[11] J. Stastny. J. Tutas. H1 internal Manual. DESY Hamburg, 1993

[12] H1 Collaboration (T. Almed tal.) Nucl Phys. B 1399 (1995) 471.

[13] J. Feltesse. Proceedings of the HERA workshop. Vol. 1. 33-58. Hamburg 1987.

[14] (i.A. Schuler and H. Spiesherger. Proceedings of the Workshop on Physics at HERA. Vol. 3: 1419-1432. Hamburg 1991

[15] A. Blondel. F. Jacquet. Procedings. Ed. I Analdi. DESY 79/48. 391-394. Hamburg 1979.

[16] A. Courau and P. Kessler. Phys. Rev. 1) (1986), 2028.

[17] HI Collaboration (I Ahmed al. 3. Z. Phys ( 666 (1995) 529.

[18] D. Blönker et al. IFEF Irans Vuel Sei, 37 (1990) 220.

[19] M. Ciluck, E. Reya and A. Vogt Physs. Lett. B306 (1993) 391.

$[20]$ A. D. Martin and $W$ J Stirling. RAL preprint RAL-93-07T (1993)

[21] A. Panitch. H1 internal note H1-08/95-449. August 1995.

[22] R. Brun et al. DID/EF/84-1. ('RRX. September 198T.

[2:3] H1 Collaboration (I Ahmed et al.). Phys Lett. B299 (1993) 374.

[24] H1 Collaboration (T. Ahmed et al.). Phys Let1. B:348 (1995) 681.

[25] H1 collaboration (s. Aidet al.) Vucl. Phys. B449 (1995) 3.

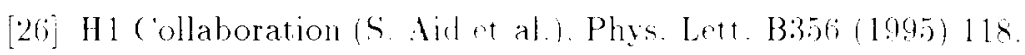

